\title{
Isolating Invisible Dynamics in the Design of Robust Hybrid Internal Models *
}

\author{
N. Cox ${ }^{\mathrm{a}, 1}$, L. Marconi $^{\mathrm{b}}$, A.R. Teel ${ }^{\mathrm{a}}$ \\ ${ }^{a}$ ECE Dept., University of California, Santa Barbara, USA \\ ${ }^{\mathrm{b}}$ C.A.SY. - DEI, University of Bologna, Bologna, Italy
}

\begin{abstract}
The paper deals with the problem of output regulation for a class of hybrid linear systems and exosystems whose state jumps periodically according to a known clock. In this framework the main contribution of the paper is to present a general method for the systematic design of robust internal model-based regulators by extending results that are known for continuous-time systems. The internal model design procedure relies upon a notion of visibility of the so-called "hybrid steady-state generator." The general theory is applied to the case of robust tracking of spline-based reference trajectories by showing how the latter can be thought of as being generated by hybrid linear exosystems.
\end{abstract}

Key words: Robust Output Regulation, Hybrid Systems, Tracking, Analysis of Systems with Uncertainties, Disturbance Rejection, Spline Trajectories

\section{Introduction}

In this paper we consider the problem of output regulation for a class of multi-input multi-output (MIMO) linear systems and exosystems that are subject to jumps according to a known clock that satisfies a periodic dwell-time constraint. Previous contributions towards hybrid output regulation leave open the crucial issue of systematically designing regulators that are robust to parametric uncertainties affecting the plant. We aim to fill this gap by addressing the issue of robustness that is normally associated with the idea of an internal model. The robustness property referred to here is what Francis and Wonham call structural stability in their milestone papers regarding output regulation and the internal model principle for continuous-time systems (see Francis \& Wonham (1975) and Francis \& Wonham (1976)).

Although this result is well-established in the continuoustime setting, in the hybrid setting some additional attention is needed to achieve robustness. We present

\footnotetext{
* Research supported by AFOSR FA9550-12-1-0127, NSF ECCS-1232035. Research supported in part by the European Project SHERPA (ICT 600958).

Email addresses: njcox@engineering.ucsb.edu (N. Cox), lorenzo.marconi@unibo.it (L. Marconi), teel@ece.ucsb.edu (A.R. Teel).

1 Corresponding author.
}

a method that is sufficient for dealing with linearly parametrized uncertainties that may affect the so-called steady-state generator. The steady-state generator is the system that generates the ideal control input, i.e. that which is able to keep the regulation error identically zero. The method builds on a "visibility property" of such a system. This property is introduced by D'Alessandro et al. (1973) in the context of a Kalmanlike decomposition for linear time-varying systems.

We design an internal model that reproduces only the "visible part" of the hybrid steady-state generator by creating an asymptotic hybrid observer of the latter. What makes the observer design interesting in the hybrid context is that there may be dynamics in the steady-state generator that are "invisible" during flows (and, as such, should not be embedded in the internal model if viewed from the continuous-time perspective) but that become "visible" during jumps, thus having an effect in achieving the regulation property. Therefore it requires a truly hybrid design in the regulator. Remarkably, this feature is particularly evident when the hybrid exosystem is chosen to reproduce spline trajectories, as such this scenario is addressed herein.

A general theory of output regulation has been presented by Marconi \& Teel (2013) for the class of singleinput single-out (SISO) linear systems and exosystems, subject to jumps according to a known clock that satis- 
fies a dwell-time constraint. They formalize the concept of steady state for hybrid systems and use it as a basic building block to develop a general theory for hybrid output regulation. Furthermore, key notions that are known for continuous-time systems, such as the regulator equations and the internal model property, are extended to that class of hybrid systems.

The design procedure presented by Marconi \& Teel (2013) relies on knowledge of the clock variable. This leads to a regulator that is linear but, in general, time varying. The case where the clock is unknown is addressed in Cox, Marconi \& Teel (2011), which leads to a regulator that, in general, is nonlinear and time-varying. Preliminary attempts to extend the theory of Marconi \& Teel (2013) to the case in which the regulated plant and exosystem are hybrid nonlinear systems have been made in Cox et al. (2013). For earlier results on designing stabilizers in conjunction with hybrid internal models see Cox et al. (2014) and Cox, Teel \& Marconi (2011). Recent relevant works in the field also include Carnevale et al. (2012a) and Carnevale et al. (2012b), regarding stabilization and regulation for MIMO, not necessarily square, hybrid linear systems. An interesting application is presented in Carnevale et al. (2013), in which the problem of tracking a spinning and bouncing disk is cast as a problem of hybrid output regulation.

The paper is laid out as follows. Section 2 details the hybrid output regulation problem and set the main framework. Section 3 presents the internal model design that is then applied in Section 4 to the case of tracking spline trajectories. Section 5 presents the practical example of robust tracking of a spline reference trajectory by a quadrotor. Some relevant propositions and proofs are saved for the appendix.

Notation The open unit disk is denoted by $\mathcal{D}_{1}$. The eigenvalues of a matrix $M$ are denoted by eig(M). The real space is denoted by $\mathbb{R}$. The Kronecker product of two matrices $A_{1}$ and $A_{2}$ is denoted by $A_{1} \otimes A_{2}$. The hybrid framework used here is presented in Goebel et al. (2009) and Goebel et al. (2012).

\section{Framework}

\subsection{Hybrid output regulation problem}

In this paper we deal with hybrid linear systems whose flow and jump relations are modeled by

$$
\begin{gathered}
\dot{\tau}=1, \dot{x}=A(\theta) x+B(\theta) u+P(\theta) w\} \\
(\tau, x, u) \in\left[0, \tau_{\max }\right] \times \mathbb{R}^{n} \times \mathbb{R}^{m} \\
\tau^{+}=0, x^{+}= \\
(\tau(\theta) x+N(\theta) w\} \\
(\tau, u) \in\left\{\tau_{\max }\right\} \times \mathbb{R}^{n} \times \mathbb{R}^{m}
\end{gathered}
$$

in which $x \in \mathbb{R}^{n}$ is the state, $u \in \mathbb{R}^{m}$ is the control input, $w$ is an exogenous variable, $\theta$ is a vector of constant un- certainties taking value in a fixed compact set $\Theta \subset \mathbb{R}^{p}$, and $\tau$ plays the role of a clock variable that is reset every $\tau_{\max }$ instances of time, with $\tau_{\max }$ representing a dwell time between two consecutive state jumps.

The exogenous variable $w$ is thought of as being generated by an exosystem that, in the proposed hybrid setting, is modeled as

$$
\begin{array}{cl}
\dot{\tau}=1, \dot{w}=S(\tau) w\} & (\tau, w) \in\left[0, \tau_{\max }\right] \times W \\
\left.\tau^{+}=0, w^{+}=J w\right\} & (\tau, w) \in\left\{\tau_{\max }\right\} \times W
\end{array}
$$

in which $W \subset \mathbb{R}^{s}$ is a compact set. In the following we assume that the set $\left[0, \tau_{\max }\right] \times W$ is forward invariant for (2). Associated with systems (1), (2), there is a regulation error $e \in \mathbb{R}^{m}$ modeled by

$$
e=C x+Q w
$$

with the triplet $(A, B, C)$ assumed to be invertible for all possible uncertainties. Namely, the matrix $C(\theta) A(\theta)^{-1} B(\theta)$ is invertible for all $\theta \in \Theta$.

The problem we are interested in is to design an error feedback hybrid regulator of the form

$$
\begin{aligned}
& \dot{\tau}=1, \dot{\eta}=F(\tau) \eta+G(\tau) e\} \quad(\tau, \eta) \in\left[0, \tau_{\max }\right] \times \mathbb{R}^{\nu} \\
& \left.\tau^{+}=0, \eta^{+}=\Phi \eta+\Sigma e\right\} \quad(\tau, \eta) \in\left\{\tau_{\max }\right\} \times \mathbb{R}^{\nu} \\
& u=\Gamma(\tau) \eta+K e
\end{aligned}
$$

so that the trajectories of the closed-loop system originating from initial conditions in $\left[0, \tau_{\max }\right] \times W \times \mathbb{R}^{n} \times \mathbb{R}^{\nu}$ and with $\theta \in \Theta$ are bounded and $\lim _{t+j \rightarrow \infty} e(t, j)=0$.

It is worth noting that the clock variable $\tau$ is assumed to be available for the design of the regulator, with the latter, in general, a time-varying system. Furthermore, it is worth emphasizing that the goal is robust regulation, namely the regulation objective must be fulfilled for all possible values of $\theta \in \Theta$. Robust tracking and/or disturbance rejection of exosystem-generated reference/disturbance signals can be clearly cast in the previous framework. Henceforth, for notational simplicity, we drop the dependence on $\theta$ from the matrices in (1).

Note that the problem of forcing the output of a continuous-time uncertain linear system to track a reference signal generated by the hybrid exosystem (2) is a simplified case that fits into the above framework. In this case, in fact, $M=I_{n}, N=0$ in (1), and $-Q w$ is the reference signal generated by (2) to be tracked by the output $C x$ of the system. This is the case considered in the example presented in Section 5.

The methods in Marconi \& Teel (2013) can be adopted to analyze the problem at hand. In Marconi \& Teel (2013), it has been shown that a necessary condition for the solution to the problem of hybrid linear regulation, as formulated above, is that there exist continuous 
functions $\Pi:\left[0, \tau_{\max }\right] \rightarrow \mathbb{R}^{n}$ and $R:\left[0, \tau_{\max }\right] \rightarrow \mathbb{R}^{m}$ that solve the Hybrid Regulator Equations

$$
\begin{aligned}
\frac{d \Pi(\tau)}{d \tau} & =A \Pi(\tau)-\Pi(\tau) S(\tau)+P+B R(\tau) \\
0 & =M \Pi\left(\tau_{\max }\right)-\Pi(0) J+N \\
0 & =C \Pi(\tau)+Q .
\end{aligned}
$$

In the following we assume that the previous equations admit a solution $(\Pi(\cdot), R(\cdot))$. The latter, since system (1) is uncertain, depends on the uncertainties $\theta$. As presented in Marconi \& Teel (2013), the existence of a solution to the hybrid regulator equations is equivalent to the existence of steady-state trajectories for the state and the input of (1) characterized by a regulation error (3) that is identically zero. In particular, with $\tau(t, j)$ and $w(t, j)$ the trajectories of (2) originating from the actual initial condition of the hybrid exosystem, it turns out that $x(t, j)=\Pi(\tau(t, j)) w(t, j)$ and $u(t, j)=R(\tau(t, j)) w(t, j)$ represent the ideal steadystate trajectory and the ideal control input toward which the state of the plant and the output of the regulator must converge.

The previous equations generalize the well-known regulator equations for continuous-time systems (Francis \& Wonham (1975) and Francis \& Wonham (1976)). The latter, indeed, are obtained from (4) considering $M=I_{n}, J=I_{s}, N=0$, a time-invariant exosystem (namely $S(\tau) \equiv S$ ), and constant unknowns $\Pi(\tau) \equiv \Pi$, $R(\tau)=R$. Under these conditions, in fact, the second equation of (4) is automatically fulfilled and the first and last equations reduce to $A \Pi-\Pi S+P+B R=0$, $C \Pi+Q=0$, which are the conventional regulator equations. Equations (4) also generalize regulator equations proposed in the context of output regulation for continuous-time linear systems in the presence of periodic exosystems (see Zhang \& Serrani (2006)).

Conditions under which the hybrid regulator equations admit a solution involve a mix of "non-resonance conditions" and "compatibility conditions" between the relative degree of the regulated plant and the hybrid exosystem. More insight into the solution and the underlying conditions can be found by expressing the triplet $(A, B, C)$ in the so-called Brunowsky canonical form. In particular, let $\left(r_{1}, \ldots, r_{m}\right)$ be the vector relative degree of the triplet $(A, B, C)$, then standard facts show that there exists a change of variable of the form $\operatorname{col}\left(z, \xi, y_{r}\right)=T x$, with $y_{r} \in \mathbb{R}^{m}, \xi \in \mathbb{R}^{r-m}$, $r=r_{1}+\ldots+r_{m}, z \in \mathbb{R}^{n-r}$, such that the flow dynamics of the regulated plant in (1) are similar to the system

$$
\begin{aligned}
\dot{z} & =A_{11} z+A_{12} L \xi+P_{1} w \\
\dot{\xi} & =A_{22} \xi+A_{23} y_{r}+P_{2} w \\
\dot{y}_{r} & =A_{31} z+A_{32} \xi+A_{33} y_{r}+\bar{B} u+P_{3} w \\
e & =L \xi+Q w
\end{aligned}
$$

where $\xi=\operatorname{col}\left(\xi_{1} \cdots \xi_{m}\right), \xi_{i}=\operatorname{col}\left(\begin{array}{lll}\xi_{i, 1} & \cdots & \xi_{i, r_{i}-1}\end{array}\right)$, $i=1, \ldots, m, A_{22}=\operatorname{blkdiag}\left(S_{1}, \ldots, S_{m}\right), A_{23}=$ $\operatorname{blkdiag}\left(B_{1}, \ldots, B_{m}\right), L=\operatorname{blkdiag}\left(L_{1}, \ldots, L_{m}\right)$, with $\left(S_{i}, B_{i}, L_{i}\right) \in \mathbb{R}^{\left(r_{i}-1\right) \times\left(r_{i}-1\right)} \times \mathbb{R}^{\left(r_{i}-1\right) \times 1} \times \mathbb{R}^{1 \times\left(r_{i}-1\right)}$ a triplet in prime form. In prime form, $S_{i}$ is a shift matrix (all 1's on the upper diagonal and all 0's elsewhere), $B_{i}^{T}=\left(\begin{array}{lll}0 \cdots 0 & 1\end{array}\right)$ and $L_{i}=(10 \cdots 0), i=1, \ldots, m . \vec{B}$ is the "high-frequency" matrix of the system (which is non-singular since $(A, B, C)$ is invertible), and $P_{1}, P_{2}$ and $P_{3}$ are appropriately defined matrices.

As for the jump dynamics, we cannot expect any special structure. In the new coordinates, the $x^{+}$expression in (1) thus takes the generic form

$$
\begin{aligned}
& z^{+}=M_{11} z+M_{12} \xi+M_{13} y_{r}+N_{1} w \\
& \xi^{+}=M_{21} z+M_{22} \xi+M_{23} y_{r}+N_{2} w \\
& y_{r}^{+}=M_{31} z+M_{32} \xi+M_{33} y_{r}+N_{3} w
\end{aligned}
$$

where $M_{i j}$ and $N_{j}$ are appropriately defined matrices.

In these coordinates the solution of the regulator equations takes a particular form. Specifically, by partitioning $\Pi(\tau)$ as $\operatorname{col}\left(\Pi_{z}(\tau), \Pi_{\xi}(\tau), \Pi_{y_{r}}(\tau)\right)$ coherently with the state partition in the new coordinates, it turns out that the first and the last equation of (4) impose that $\Pi_{\xi}(\tau)$ is necessarily given by

$$
\Pi_{\xi}(\tau)=\left(\begin{array}{c}
\Pi_{\xi_{1}}(\tau) \\
\vdots \\
\Pi_{\xi_{m}}(\tau)
\end{array}\right), \quad \Pi_{\xi_{i}}(\tau)=\left(\begin{array}{c}
\Pi_{\xi_{i, 1}}(\tau) \\
\vdots \\
\Pi_{\xi_{i, r_{i}-1}(\tau)}
\end{array}\right)
$$

where the $\Pi_{\xi_{i, j}}(\tau)$ are recursively defined as

$$
\begin{aligned}
\Pi_{\xi_{i, 1}} & =-Q_{i} \\
\Pi_{\xi_{i, j}}(\tau) & =\frac{d \Pi_{\xi_{i, j-1}}(\tau)}{d \tau}+\Pi_{\xi_{i, j-1}}(\tau) S(\tau)-P_{2 i, j-1}
\end{aligned}
$$

for $i=1, \ldots, m, j=2, \ldots, r_{i}-1$, in which $P_{2 i, j}$ represents the $j$-th rows of $P_{2 i}$ having partitioned $P_{2}$ as $\operatorname{col}\left(P_{21}, \ldots, P_{2 m}\right)$ coherently with the partition of $\xi$. Furthermore, by the first equation of $(4), \Pi_{y_{r}}(\tau)$ is necessarily given by

$$
\Pi_{y_{r}}(\tau)=\frac{d \Pi_{\xi_{i, r_{i}-1}}(\tau)}{d \tau}+\Pi_{\xi_{i, r_{i}-1}}(\tau) S(\tau)-P_{2 i, r_{i}-1} .
$$

The previous definitions of $\Pi_{\xi}$ and $\Pi_{y_{r}}$, in fact, guarantee

$$
\begin{aligned}
\frac{d \Pi_{\xi}(\tau)}{d \tau} & =A_{22} \Pi_{\xi}(\tau)+A_{23} \Pi_{y_{r}}(\tau)-\Pi_{\xi}(\tau) S(\tau)+P_{2} \\
0 & =L \Pi_{\xi}(\tau)+Q .
\end{aligned}
$$


With $\Pi_{\xi}(\tau)$ and $\Pi_{y_{r}}(\tau)$ fixed, the remaining unknowns $\Pi_{z}(\tau)$ and $R(\tau)$ result from the first two equations in (4). In particular, $\Pi_{z}(\tau)$ is constrained to be a solution of

$$
\begin{aligned}
\frac{d \Pi_{z}(\tau)}{d \tau}= & A_{11} \Pi_{z}(\tau)-A_{12} Q-\Pi_{z}(\tau) S+P_{1} \\
0= & M_{11} \Pi_{z}\left(\tau_{\max }\right)-\Pi_{z}(0) J \\
& +M_{12} \Pi_{\xi}\left(\tau_{\max }\right)+M_{13} \Pi_{y_{r}}\left(\tau_{\max }\right)+N_{1}
\end{aligned}
$$

with the additional constraints

$$
\begin{aligned}
& \left(\begin{array}{c}
M_{21} \\
M_{31}
\end{array}\right) \Pi_{z}\left(\tau_{\max }\right)-\left(\begin{array}{c}
\Pi_{\xi}(0) \\
\Pi_{y_{r}}(0)
\end{array}\right) J+\left(\begin{array}{c}
N_{2} \\
N_{3}
\end{array}\right) \\
& +\left(\begin{array}{c}
M_{22} \\
M_{32}
\end{array}\right) \Pi_{\xi}\left(\tau_{\max }\right)+\left(\begin{array}{c}
M_{23} \\
M_{33}
\end{array}\right) \Pi_{y_{r}}\left(\tau_{\max }\right)=0 .
\end{aligned}
$$

With $\Pi_{z}(\tau)$ also in hand, $R(\tau)$ is then determined by

$$
\begin{gathered}
R(\tau)=\bar{B}^{-1}\left(\frac{d \Pi_{y_{r}}(\tau)}{d \tau}+\Pi_{y_{r}}(\tau) S(\tau)-A_{31} \Pi_{z}(\tau)\right. \\
\left.-A_{32} \Pi_{\xi}(\tau)-A_{33} \Pi_{y_{r}}(\tau)-P_{3}\right) .
\end{gathered}
$$

The solution of the hybrid regulator equations thus boils down to computing a $\Pi_{z}(\tau)$ fulfilling (10) and the additional constraints (11). In this respect the following result, proved in (Marconi \& Teel 2013, Lemma 1), details a "non-resonance condition" under which a $\Pi_{z}(\tau)$ fulfilling (10) exists.

Lemma 1 Let $\phi_{S}(\tau)$ be the state transition matrix of the time-varying system $\dot{w}=S(\tau) w$, namely the smooth matrix such that $d \phi_{S}(\tau) / d \tau=S(\tau) \phi_{S}(\tau)$ and $\phi_{S}(0)=$ $I_{s}$. Assume that the "non-resonance condition"

$$
\operatorname{eig}\left(M_{11} \exp \left(A_{11} \tau_{\max }\right)\right) \cap \operatorname{eig}\left(J \phi_{S}\left(\tau_{\max }\right)\right)=\emptyset
$$

holds true. Then for all $\Pi_{\xi}\left(\tau_{\max }\right)$ and $\Pi_{y_{r}}\left(\tau_{\max }\right)$ there exists a unique continuously differentiable solution $\Pi_{z}(\tau)$ to the equations (10). In fact, the solution is

$$
\begin{aligned}
\Pi_{z}(\tau)=\left(\exp \left(A_{11} \tau\right) Z+\right. & \\
& \left.\int_{0}^{\tau} \exp \left(A_{11}(t-\tau)\right) \bar{G} \phi_{S}(t) d t\right) \phi_{S}(-\tau)
\end{aligned}
$$

with $Z$ the solution of

$$
M_{11} \exp \left(A_{11} \tau_{\max }\right) Z-Z J \phi_{S}\left(\tau_{\max }\right)+\bar{H}=0
$$

where $\bar{G}=P_{1}-A_{12} Q$ and $\bar{H}=N \phi_{S}\left(\tau_{\max }\right)+$ $M_{11} \int_{0}^{\tau_{\max }} \exp \left(A_{11}\left(t-\tau_{\max }\right)\right) \bar{G} \phi_{S}(t) d t$.
As the eigenvalues of $A_{11}$ coincide with the transmission zeros of the triplet $(A, B, C)$, condition (13) can be interpreted as the generalization, in the considered hybrid setting, of the non-resonance condition between the zeros of the controlled plant and the modes of the exosystem, which is a well-known condition in the continuoustime domain.

With $\Pi_{z}(\cdot)$ also given, the hybrid regulator equations (4) are fulfilled if $\left(\Pi_{z}(\cdot), \Pi_{\xi}(\cdot), \Pi_{y_{r}}(\cdot)\right)$ are such that the constraints (11) are satisfied. Those constraints can be regarded as "compatibility constraints" between the relative degree of the regulated plant and the hybrid exosystem. It essentially fixes a requirement on the continuity of the "reference signal" $Q w(t)$ during jumps in relation to the relative degree of the system.

For example, in the case where the plant is a continuoustime system with unitary relative degree (namely $r_{i}=1$, $i=1, \ldots, m)$, it is immediately seen that (11) are automatically fulfilled if $Q J=Q$. Namely, if $(Q w)^{+}=Q w$, which is a requirement on the continuity of the output reference signal during jumps.

Henceforth we consider such "compatibility constraints" fulfilled, which implies that the hybrid regulator equations have a solution. The latter is given by $\Pi_{\xi}(\cdot)$ and $\Pi_{y_{r}}(\cdot)$ in $(6),(7),(8)$, by $\Pi_{z}(\cdot)$ given by (14)-(15), and by $R(\cdot)$ in $(12)$. We make such a requirement explicit in the following assumption.

Assumption 1 For all $\theta$ in $\Theta$ such that the "nonresonance" condition (13) holds, the unique solution of (6)-(10) satisfies the compatibility constraint (11).

We complete the section by presenting two assumptions under which the problem of hybrid output regulation is solved. The first asks that the hybrid system is minimum phase as detailed in Assumption 2 below. This assumption is not necessary and is only formulated in order to present a systematic robust design procedure for the regulator based on high-gain arguments. The second assumption is made in order to achieve the stabilization goal using a continuous-time high-gain observer (see Theorem 1). It is conceivable that if a different, possibly hybrid, stabilizer is used in order to achieve hybrid output regulation, then this assumption can be removed. Furthermore, it is worth noting that this assumption comes into play only in the presence of systems without a unitary relative degree vector.

Assumption 2 (Minimum Phase) The matrices $A_{11}, M_{11}$ are such that $\operatorname{eig}\left(M_{11} \exp \left(A_{11} \tau_{\max }\right)\right) \in \mathcal{D}_{1}$.

Assumption 3 The matrix parameters $M_{i j}$ are such that $M_{i j}=I$ if $i=j$ and $M_{i j}=0$ otherwise.

\subsection{Hybrid steady-state generator system}

A crucial role in the design of internal model-based regulators is played by the so-called (see Marconi \& Teel 
(2013)) "hybrid steady-state generator system" defined as the following hybrid system

$$
\begin{array}{ll}
\dot{\tau}=1, \dot{w}=S(\tau) w\} & (\tau, w) \in\left[0, \tau_{\max }\right] \times W \\
\left.\tau^{+}=0, w^{+}=J w\right\} & (\tau, w) \in\left\{\tau_{\max }\right\} \times W \\
y_{w}=R(\tau) w &
\end{array}
$$

with output $y_{w} \in \mathbb{R}^{m}$. In fact, this system generates all the ideal steady-state control inputs required by the regulator in order to keep the regulation error $e$ identically zero. Due to the fact that the initial condition of the exosystem is arbitrary on $\left[0, \tau_{\max }\right] \times W$, it is apparent that the "visible" dynamics of system (16) must be embedded into any regulator that solves the problem of output regulation ${ }^{2}$. This observation is at the foundation of the celebrated internal model principle (see Francis \& Wonham (1976) for continuous-time linear systems and Marconi \& Teel (2013) for hybrid linear systems).

An obstacle to embedding a copy of (16) into the regulator stems from the fact that the function $R(\cdot)$, as a solution of the hybrid regulator equations is, in general, affected by the parametric uncertainties $\theta$ in the regulated plant. This fact raises an issue of robust design of the regulator. In order to deal with this issue it is worth introducing the class of systems that are "state-output" equivalent to (16) as formally defined in Definition 1 below. In the definition we refer to an "equivalent" system defined by

$$
\begin{array}{cc}
\dot{\tau}=1, \dot{\mathbf{w}}=\mathbf{S}(\tau) \mathbf{w}\} & (\tau, \mathbf{w}) \in\left[0, \tau_{\max }\right] \times \mathbf{W} \\
\left.\tau^{+}=0, \mathbf{w}^{+}=\mathbf{J} \mathbf{w}\right\} & (\tau, \mathbf{w}) \in\left\{\tau_{\max }\right\} \times \mathbf{W} \\
\mathbf{y}_{\mathbf{w}}=\mathbf{R}(\tau) \mathbf{w} &
\end{array}
$$

where $\mathbf{w} \in \mathbb{R}^{\mathbf{s}}, \mathbf{y}_{\mathbf{w}} \in \mathbb{R}^{m}, \mathbf{s} \in \mathbb{N}$, and $\mathbf{W}$ is a compact subset of $\mathbb{R}^{\mathbf{s}}$ with $\left[0, \tau_{\max }\right] \times \mathbf{W}$ invariant for $(17)$. We note that (16) and (17) have the same hybrid time domain (see Goebel et al. (2009)), which is dependent on the initial value of $\tau$.

Definition 1 System (16) is state-output equivalent to system (17) if for each solution of (16) there exists a solution of (17) with the same hybrid time domain, which we denote by $E$, and $y_{w}(t, j)=\mathbf{y}_{\mathbf{w}}(t, j)$ for all $(t, j) \in E$.

The idea behind the design of robust regulators is to embed a copy of a state-output equivalent system of (16) that is not affected by uncertainties. This is easy to achieve in the context of continuous-time linear regulation (Francis \& Wonham $(1975,1976))$, in which the

\footnotetext{
2 The concept of visibility is used loosely here, but is better specified later by following D'Alessandro et al. (1973). Intuitively, visible dynamics are state trajectories of (16) that show up on the output $y_{w}$ and, as such, must be reproduced by the regulator.
}

steady-state generator system takes the form $\dot{w}=S w$, $y_{w}=R w$. As a matter of fact, denoting by $d$ and by $a_{i}, i=1, \ldots, d$, the order and the coefficients of the minimal polynomial of $S$, respectively, it turns out that a state-output equivalent system $\dot{\mathbf{w}}=\mathbf{S w}, \mathbf{y}_{\mathbf{w}}=\mathbf{R} \mathbf{w}$ can be obtained by taking

$$
\begin{gathered}
\mathbf{S}=\left(\begin{array}{ccccc}
0 & I_{m} & 0 & \ldots & 0 \\
0 & 0 & I_{m} & \ldots & 0 \\
\cdot & \cdot & \cdot & \ldots & . \\
0 & 0 & 0 & \ldots & I_{m} \\
-a_{1} I_{m} & -a_{2} I_{m} & -a_{3} I_{m} & \ldots & -a_{d} I_{m}
\end{array}\right), \\
\mathbf{R}=\left(\begin{array}{cccc}
I_{m} & 0 & 0 & \ldots
\end{array}\right) .
\end{gathered}
$$

With such a choice, in fact, any output behavior $y_{w}(t)$ of $\dot{w}=S w, y_{w}=R w$, originating from an arbitrary initial condition $w(0)$, can be generated as the output behavior $\mathbf{y}_{\mathbf{w}}(t)$ of $\dot{\mathbf{w}}=\mathbf{S w}$, $\mathbf{y}_{\mathbf{w}}=\mathbf{R} \mathbf{w}$ with initial condition $\mathbf{w}(0)=\Sigma w(0)$, where $\Sigma=\left[\begin{array}{llll}R^{T} & S^{T} R^{T} & \ldots & S^{(d-1) T} R^{T}\end{array}\right]^{T}$. Namely, the pairs $(S, R)$ and $(\mathbf{S}, \mathbf{R})$ are state-output equivalent, with the clear benefit of the latter being independent of possible uncertainties entering into $R$.

The case of hybrid systems, on the other hand, is more involved for at least two reasons. First, the matrices $S(\cdot)$ and $R(\cdot)$ are, in general, time varying since they depend on the clock. Furthermore, the state-output equivalence must be guaranteed not only during flows but also during jumps. These peculiarities justify a different approach to the problem, as presented below.

In the proposed approach we assume that $R(\tau)$ is linearly parametrized in the uncertainties. In particular, by partitioning $R(\cdot)$ input-wise as $R(\cdot)=\operatorname{col}\left(R_{1}(\cdot), \ldots, R_{m}(\cdot)\right)$ with $R_{i}:\left[0, \tau_{\max }\right] \rightarrow \mathbb{R}^{1 \times s}$, we assume the following.

Assumption 4 There exist $p_{i} \in \mathbb{N}$ and known continuously differentiable functions $R_{i j}:\left[0, \tau_{\max }\right] \rightarrow \mathbb{R}^{1 \times s}$, $i=1, \ldots, m, j=1, \ldots, p_{i}$, such that

$$
R_{i}(\tau)=\sum_{j=1}^{p_{i}} R_{i j}(\tau) \mu_{i j}
$$

where $\mu_{i j} \in \mathbb{R}$ are uncertain parameters ranging in a known compact set.

Under this condition, a hybrid steady-state generator equivalent to (16), but unaffected by uncertainties, can be constructed as detailed in the next proposition (whose proof comes by slightly adapting the arguments of the proof of Proposition 6 in Marconi \& Teel (2013)).

Proposition 1 With $p=\sum_{i=1}^{m} p_{i}, \mathbf{R}_{i}(\tau)=\left(R_{i 1}(\tau), \ldots\right.$, $\left.R_{i, p_{i}}(\tau)\right), i=1, \ldots, m$, and $W_{i j}=\left\{\bar{w} \in \mathbb{R}^{s}: \bar{w}=\right.$ 
$\left.\mu_{i j} w, w \in W\right\}, i=1, \ldots, m, j=1, \ldots, p_{i}$, let

$$
\begin{aligned}
\mathbf{S}(\tau) & =I_{p} \otimes S(\tau), \quad \mathbf{J}=I_{p} \otimes J \\
\mathbf{R}(\tau) & =\operatorname{blockdiag}\left(\mathbf{R}_{1}(\tau), \ldots, \mathbf{R}_{m}(\tau)\right) \\
\mathbf{W} & =W_{11} \times \ldots \times W_{1 p_{1}} \times \ldots \times W_{m 1} \times \ldots \times W_{m p_{m}} .
\end{aligned}
$$

Then system (17) is state-output equivalent to (16).

By bearing in mind the expression of $R(\cdot)$ in (12) as a function of $\Pi_{y_{r}}(\cdot), \Pi_{\xi}(\cdot)$, and $\Pi_{z}(\cdot)$, and the dependence of the latter on $A_{11}$ given in (14), it is immediately seen that $R(\tau)$ is linearly parametrized in the uncertainties whenever the natural modes of the flow zero dynamics (namely the eigenvalues of the matrix $A_{11}$ ) are independent of parameter variations.

\subsection{Main result about regulator design}

In this section we present a general result on the design of an internal-model based regulator. Such a result builds on a notion of the Hybrid Internal Model Property that is precisely defined in the following.

Definition 2 (Hybrid Internal Model Property) A quadruplet $\left(F_{\mathrm{im}}(\cdot), G_{\mathrm{im}}(\cdot), \Gamma_{\mathrm{im}}(\cdot), \Sigma_{\mathrm{im}}\right)$, where $F_{\mathrm{im}}$ : $\left[0, \tau_{\max }\right] \rightarrow \mathbb{R}^{\nu \times \nu}, G_{\mathrm{im}}:\left[0, \tau_{\max }\right] \rightarrow \mathbb{R}^{\nu \times m}$ and $\Gamma_{\mathrm{im}}:$ $\left[0, \tau_{\max }\right] \rightarrow \mathbb{R}^{1 \times \nu}, \nu \in \mathbb{N}$ are continuously differentiable functions and $\Sigma_{\mathrm{im}}$ is a matrix, has the hybrid internal model property relative to (17) if for some continuously differentiable function $\Pi_{\eta}:\left[0, \tau_{\max }\right] \rightarrow \mathbb{R}^{\nu \times \mathbf{s}}$ the set

$$
\mathcal{S}=\left\{(\tau, \mathbf{w}, \eta) \in\left[0, \tau_{\max }\right] \times \mathbf{W} \times \mathbb{R}^{\nu}: \eta=\Pi_{\eta}(\tau) \mathbf{w}\right\}
$$

is globally exponentially stable for the hybrid system

$$
\begin{aligned}
& \left.\begin{array}{l}
\dot{\tau}=1, \dot{\mathbf{w}}=\mathbf{S}(\tau) \mathbf{w} \\
\dot{\eta}=F_{\mathrm{im}}(\tau) \eta+G_{\mathrm{im}}(\tau) \mathbf{R}(\tau) \mathbf{w}
\end{array}\right\} \\
& (\tau, \mathbf{w}, \eta) \in\left[0, \tau_{\max }\right] \times \mathbf{W} \times \mathbb{R}^{\nu} \\
& \left.\begin{array}{l}
\tau^{+}=0, \mathbf{w}^{+}=\mathbf{J} \mathbf{w} \\
\eta^{+}=\Sigma_{\mathrm{im}} \eta
\end{array}\right\} \\
& \begin{aligned}
&(\tau, \mathbf{w}, \eta) \in\left\{\tau_{\max }\right\} \times \mathbf{W} \times \mathbb{R}^{\nu} \\
& \text { and } \quad \Gamma_{\mathrm{im}}(\tau) \Pi_{\eta}(\tau)=\mathbf{R}(\tau) \quad \forall \tau \in\left[0, \tau_{\max }\right]
\end{aligned}
\end{aligned}
$$

It is worth noting that if a quadruplet $\left(F_{\mathrm{im}}(\cdot), G_{\mathrm{im}}(\cdot)\right.$, $\left.\Gamma_{\mathrm{im}}(\cdot), \Sigma_{\mathrm{im}}\right)$ has the hybrid internal model property then the set (19) is forward invariant for the hybrid system (20). In this case the function $\Pi_{\eta}(\tau)$ necessarily satisfies the equations (Marconi \& Teel (2013))

$$
\begin{aligned}
\frac{d \Pi_{\eta}(\tau)}{d \tau} & =F_{\mathrm{im}}(\tau) \Pi_{\eta}(\tau)-\Pi_{\eta}(\tau) \mathbf{S}+G_{\mathrm{im}}(\tau) \mathbf{R}(\tau) \\
0 & =\Sigma_{\mathrm{im}} \Pi_{\eta}\left(\tau_{\max }\right)-\Pi_{\eta}(0) \mathbf{J}
\end{aligned}
$$

that, in turn, express the forward invariance of (19) both during flows and during jumps.
Later in this section and more extensively in Section 3, a systematic procedure for designing quadruplets with the hybrid internal model property is presented. For the time being we show that if such a quadruplet exists then a regulator solving the problem at hand can be systematically designed. Toward this end, by following the prescriptions of Marconi \& Teel (2013), we consider a hybrid internal model-based regulator of the form

$$
\begin{aligned}
& \dot{\tau}=1, \dot{\eta}=F_{\mathrm{im}}(\tau) \eta+G_{\mathrm{im}}(\tau) u \\
& \dot{\chi}=\Phi_{\mathrm{st}} \chi+\Lambda_{\mathrm{st}}(L \xi+Q w) \\
& (\tau, \eta, \chi) \in\left[0, \tau_{\max }\right] \times \mathbb{R}^{\nu} \times \mathbb{R}^{r} \\
& \left.\tau^{+}=0, \eta^{+}=\Sigma_{\mathrm{im}} \eta, \chi^{+}=\chi\right\} \\
& (\tau, \eta, \chi) \in\left\{\tau_{\max }\right\} \times \mathbb{R}^{\nu} \times \mathbb{R}^{r} \\
& \begin{array}{c}
u=\Gamma_{\mathrm{im}}(\tau) \eta-\kappa \bar{B}^{-1} K \chi
\end{array}
\end{aligned}
$$

where $K=\operatorname{blkdiag}\left(K_{1}, \ldots, K_{m}\right)$, with $K_{i}=\left(k_{i 1} \ldots\right.$ $\left.k_{i r_{i}-1} 1\right)$ such that the $k_{i j}$ are coefficients of a Hurwitz polynomial $s^{r_{i}-1}+k_{i 1} s^{r_{i}-2}+\cdots+k_{i r_{i}-1}$, and $\kappa$ is a design parameter. The matrix parameters $\Phi_{\text {st }}$ and $\Lambda_{\text {st }}$ are chosen as block diagonal matrices, with the blocks

$\Lambda_{\mathrm{st}_{i}}=\left(\begin{array}{lll}c_{i 1} g & \cdots & c_{i r_{i}} g^{r_{i}}\end{array}\right)^{\mathrm{T}}, \quad \Phi_{\mathrm{st}_{i}}=\left(\begin{array}{cc} & I_{r_{i}-1} \\ -\Lambda_{\mathrm{st}_{i}} & 0\end{array}\right)$

with $g$ a design parameter and $c_{i j}$ coefficients of a Hurwitz polynomial.

Note that the stabilizer unit of the regulator takes the form of a high-gain observer, which is appropriate due to its robustness to parameter variation in the plant. It is also worth noting that the design of the stabilizer is not the main point of the paper. It is perfectly conceivable that the stabilization unit could be designed using a different scheme, while still using the robust internal model design results presented in the following sections. The following result, whose proof is in Appendix A, provides the main guidelines for the design of (23).

Theorem 1 Let Assumptions 1, 2, 3 and 4 be satisfied. Let (17) be a system that is state-output equivalent to the hybrid steady-state generator system (16) and let the quadruplet $\left(F_{\mathrm{im}}(\cdot), G_{\mathrm{im}}(\cdot), \Gamma_{\mathrm{im}}(\cdot), \Sigma_{\mathrm{im}}\right)$ have the hybrid internal model property relative to (17). Then there exists $a \kappa^{*}>0$ and for each $\kappa \geq \kappa^{*}$ there exists a $g^{*}>0$ such that for each $g \geq g^{*}$ the regulator (23) solves the problem of robust hybrid output regulation.

In the control structure (23) one immediately recognizes an internal model unit, with state $\eta$, and a stabilizing unit with state $\chi$. The former is designed by following the properties of Definition 2. The latter, on the other hand, relies upon a high-gain observer structure by taking advantage of the minimum-phase assumption (see 
the proof of Theorem 1). For systems having unitary vector relative degree such a stabilizer is static. In particular, in such a case, the arguments used in the proof of the proposition show that the control law can be taken as $u=\Gamma_{\mathrm{im}}(\tau) \eta+\kappa \bar{B}^{-1} H e$ with $H \in \mathbb{R}^{m \times m}$ a Hurwitz matrix and $\kappa$ a sufficiently large gain.

As is clear from the statement of Definition 2, the design of the internal model unit entails the design of an asymptotic output reproducer for a hybrid system that is stateoutput equivalent to the hybrid steady-state generator (16). Namely, the goal is to design a hybrid system that, forced by $\mathbf{y}_{\mathbf{w}}$, is able to asymptotically reproduce all of the possible output behaviors $\mathbf{y}_{\mathbf{w}}(t, j)$ of $(17)$, and thus all of the possible output behaviors $y_{w}(t, j)$ of $(16)$. Although the problem is intimately related to a problem of state observation, it is worth noting how, in the output regulation context, the main issue is not to estimate the state $\mathbf{w}$, but rather to reproduce the output $\mathbf{y}_{\mathbf{w}}$. In this respect, system (17) is not, in principle, required to have all the dynamics that are "visible". State components of (17) that are eventually "invisible," need not, in principle, be reproduced in the internal model, as they are not essential in achieving the output reproducer capabilities mentioned above.

\section{Achieving the Hybrid Asymptotic Internal Model Property}

In this section we address the problem of systematically designing a quadruplet $\left(F_{\mathrm{im}}(\tau), G_{\mathrm{im}}(\tau), \Gamma_{\mathrm{im}}(\tau), \Sigma_{\mathrm{im}}\right)$ that has the hybrid internal model property according to Definition 2. We start by recalling a result presented in (Marconi \& Teel 2013, Proposition 4).

Proposition 2 Let $\nu \geq \mathbf{s}$ and $\left(F_{\mathrm{im}}, G_{\mathrm{im}}, \Sigma_{\mathrm{im}}\right)$ be chosen such that the pair $\left(F_{\mathrm{im}}, G_{\mathrm{im}}\right)$ is controllable, $\operatorname{eig}\left(\Sigma_{\operatorname{im}} \exp \left(F_{\text {im }} \tau_{\max }\right)\right) \cap \operatorname{eig}\left(J \phi_{\mathbf{S}}\left(\tau_{\max }\right)\right)=\emptyset$, and $\Sigma_{\mathrm{im}} \exp \left(F_{\mathrm{im}} \tau_{\max }\right) \in \mathcal{D}_{1}$. Then there exists a unique continuously differentiable function $\Pi_{\eta}:\left[0, \tau_{\max }\right] \rightarrow \mathbb{R}^{\nu \times \mathbf{s}}$ that is the solution of (22) such that the set $\mathcal{S}$ in (19) is globally exponentially stable for (20). Furthermore, the function $\Gamma_{\mathrm{im}}(\tau)=\mathbf{R}(\tau) \Pi_{\eta}^{\dagger}(\tau)$, where $\Pi_{\eta}^{\dagger}(\tau)$ is the Moore-Penrose pseudo-inverse of $\Pi_{\eta}(\tau)$, is such that (21) is fulfilled. Namely, the quadruplet $\left(F_{\mathrm{im}}, G_{\mathrm{im}}, \Gamma_{\mathrm{im}}(\tau), \Sigma_{\mathrm{im}}\right)$ has the hybrid internal model property.

The existence of a continuously differentiable function $\Pi_{\eta}(\tau)$ that solves (22) follows from Marconi \& Teel (2013) by using the fact that $\operatorname{eig}\left(\Sigma_{\mathrm{im}} \exp \left(F_{\mathrm{im}} \tau_{\max }\right)\right) \in$ $\mathcal{D}_{1}$ and $\operatorname{eig}\left(\Sigma_{\mathrm{im}} \exp \left(F_{\mathrm{im}} \tau_{\max }\right)\right) \cap \operatorname{eig}\left(J \phi_{\mathbf{S}}\left(\tau_{\max }\right)\right)=\emptyset$. The fact that $\nu \geq \mathbf{s}$ and the pair $\left(F_{\mathrm{im}}, G_{\mathrm{im}}\right)$ is controllable plays a role in proving that $\Gamma_{\mathrm{im}}(\tau)=R(\tau) \Pi_{\eta}^{\dagger}(\tau)$ fulfills (21), see Proposition 4 in Marconi \& Teel (2010).

Although the previous result shows that a quadruplet with the asymptotic hybrid internal model property, and thus an error feedback regulator, can always be designed, it is not conclusive about the fact that such a regulator is continuous. As a matter of fact, there is no guarantee that $\Pi_{\eta}^{\dagger}(\tau)$, and thus $\Gamma_{\mathrm{im}}(\tau)$, is continuous. By following Stewart (1969), it turns out that a sufficient condition under which the function $\Pi_{\eta}^{\dagger}(\tau)$ is continuous is the existence of an $r \leq \mathbf{s}$ such that $r=\operatorname{rank}\left(\Pi_{\eta}(\tau)\right)$ for all $\tau \in\left[0, \tau_{\max }\right]$. The fulfillment of such a sufficient condition is, in general, affected by all the matrices entering into (22), among which the pair $(S, J)$ define the hybrid exosystem. A relevant case that does not fulfill such a condition is presented in Section 4 , in which the hybrid exosystem is chosen to generate a spline signal. In all the cases in which the methodology presented in Proposition 2 does not yield a continuous regulator, a different design methodology should be explored. This is done in the remainder of the section.

We approach the problem of designing a quadruplet $\left(F_{\mathrm{im}}(\tau), G_{\mathrm{im}}(\tau), \Gamma_{\mathrm{im}}(\tau), \Sigma_{\mathrm{im}}\right)$ fulfilling the properties of Definition 2 by designing an observer for the dynamics of (17) that are "visible" on the output $\mathbf{y}_{\mathbf{w}}$. Toward this end, in the next subsection we present a decomposition of system (17) that isolates visible and invisible dynamics. Our goal is to identify a hybrid system that is stateoutput equivalent to (17) and for which an asymptotic observer can be designed. The design of the (hybrid) asymptotic observer is dealt with in Section 3.2. This, in turn, leads to immediately obtaining an "output reproducer" of system (17). For notational convenience, in the following part we drop the bold notation for system (17) by using $S(\tau), J, R(\tau), W$ and $y_{w}$ instead of $\mathbf{S}(\tau), \mathbf{J}, \mathbf{R}(\tau), \mathbf{W}$ and $\mathbf{y}_{\mathbf{w}}$.

\subsection{Isolating invisible dynamics}

Towards the final goal of isolating visible and invisible dynamics of the hybrid system (17), we start by focusing on the flow dynamics by identifying dynamics that do not affect the output during flow. Consider the continuous-time time-varying linear system of the form

$$
\dot{w}=S(\tau) w, \quad y_{w}=R(\tau) w \quad w \in \mathbb{R}^{s},
$$

defined on the interval $\tau \in\left[0, \tau_{\max }\right]$ and let $\phi_{S}\left(\tau, \tau_{0}\right)$ be the state transition matrix associated with $\dot{w}=S(\tau) w$. As the system is time-varying, a Kalman-like decomposition related to observability can be rigorously obtained by the arguments of D'Alessandro et al. (1973). The definition of an invisible state is crucial to that paper and is recalled here properly adapted to our purposes.

Definition 3 We say that a state $w \in \mathbb{R}^{s}$ is invisible at time $\tau \in\left[0, \tau_{\max }\right]$ in the interval $\left[0, \tau_{\max }\right]$ if it is unobservable and unreconstructable at time $\tau$ in the specified time interval, namely if

$$
R(t) \phi_{S}(t, \tau) w=0 \quad \text { for all } t \in\left[0, \tau_{\max }\right] .
$$

Note that the previous relation holds for all $t$ in the interval, namely for $t \geq \tau$ (unobservability of the state 
$w$ ) and for $t \leq \tau$ (unreconstructability of the state $w$ ). In other words, all the output behavior, $y_{w}(t)$, originating from an invisible state at time $\tau$ is identically zero (in the interval) both forward (i.e. for $t \geq \tau$ ) and backward $(t \leq \tau)$ in time. The arguments in D'Alessandro et al. (1973) can be used off-the-shelf in order to find a change of variable that isolates the invisible states of the flow dynamics of (17). For the sake of compactness we present the main steps here, while linking the reader interested in details to D'Alessandro et al. (1973).

Let $\mathcal{G}$ be the Gramian at time 0 associated with the system in the interval defined as

$$
\mathcal{G}=\int_{0}^{\tau_{\max }} \phi_{S}(t, 0)^{T} R^{T}(t) R(t) \phi_{S}(t, 0) d t
$$

and let $s_{n o}=\operatorname{dimKer}(\mathcal{G})$. Furthermore, let $\left\{\mathrm{v}_{i}\right\}_{i=1}^{s_{n o}}$ and $\left\{\mathrm{v}_{i}\right\}_{i=s_{n_{o}}+1}^{s}$, respectively, be bases of $\operatorname{Ker}(\mathcal{G})$ and $\operatorname{Im}(\mathcal{G})$, and consider the change of variables $w=T(\tau) \varsigma$ with

$$
T(\tau)=\phi_{S}(\tau, 0) V, V:=\left[\mathrm{v}_{1} \cdots \mathrm{v}_{s_{n o}} \mathrm{v}_{s_{n o}+1} \cdots \mathrm{v}_{s}\right] .
$$

Following D'Alessandro et al. (1973), and partitioning $\varsigma=\operatorname{col}\left(\varsigma_{o}, \varsigma_{\text {no }}\right)$ consistently with the partition of $T(\cdot)$, system (24) in the new coordinates reads as

$$
\dot{\varsigma}_{\mathrm{no}}=0, \quad \dot{\varsigma}_{\mathrm{o}}=0, \quad y_{w}=R_{\mathrm{o}}(\tau) \varsigma_{\mathrm{o}},
$$

with $\varsigma_{n o} \in \mathbb{R}^{s_{n o}}, \varsigma_{o}=\mathbb{R}^{s_{o}}$, where $s_{o}:=s-s_{n o}$ and

$$
R_{o}(\tau)=R(\tau) \phi_{S}(\tau, 0)\left[\mathrm{v}_{s_{n o}+1} \cdots \mathrm{v}_{s}\right],
$$

with the Gramian

$$
\mathcal{G}_{o}=\int_{0}^{\tau_{\max }} R_{o}^{T}(t) R_{o}(t) d t
$$

associated with the $\varsigma_{o}$ subsystem that is not singular.

We note that the subspace $\left\{\varsigma: \varsigma_{o}=0\right\}$ is invariant and composed of invisible states, whereas the subsystem $\dot{\varsigma}_{o}=0$ with output $y_{w}=R_{o}(\tau) \varsigma_{o}$ is "visible" in the interval.

Remark 1 It is worth noting that the previous visibility property of the pair $\left(0, R_{o}(\cdot)\right)$ does not imply, in general, that the pair is uniformly observable, i.e. that the observability matrix associated with the pair $\left(0, R_{o}(\tau)\right)$ is nonsingular for all $\tau$ in the interval. The latter property is, in fact, related to a uniform observability that is time-wise.

We now consider the hybrid system (17), with the goal of identifying visible and invisible dynamics for this system. By applying the change of variable $T(\tau)$ discussed above, the jump relation of system (17) transforms as

$\varsigma^{+}=\left[T\left(\tau_{\max }\right)^{-1}\right]^{+} w^{+}=T(0)^{-1} J w=T(0)^{-1} J T\left(\tau_{\max }\right) \varsigma$.
By partitioning $T(0)^{-1} J T\left(\tau_{\max }\right)$ consistently with $\varsigma$, we obtain

$$
\varsigma_{o}^{+}=J_{o} \varsigma_{o}+J_{\text {ono }} \varsigma_{n o}, \quad \varsigma_{n o}^{+}=J_{n o} \varsigma_{n o}+J_{n o o} \varsigma_{o},
$$

where the matrices $J_{o}, J_{\text {ono }}, J_{\text {no }}, J_{\text {noo }}$ do not have any special properties.

By construction, the hybrid system flowing according to (26) and jumping according to (28) is state-output equivalent to (17). Furthermore, the $\varsigma_{\text {no }}$ state component, that is invisible for the continuous-time system (24) during flows, might become visible for the hybrid system (17). As a matter of fact, the $\varsigma_{n o}$ component might show up during jumps by affecting $\varsigma_{o}$ through the jump relation $\varsigma_{o}^{+}=J_{o} \varsigma_{o}+J_{o n o} \varsigma_{n o}$, thus affecting the output $y_{w}(t)$ in the "subsequent" flow interval. This means that, in the attempt to identify a system that is state-output equivalent to (17) and for which an asymptotic observer can be designed, it cannot be ignored.

This observation motivates the forthcoming developments, in which the goal is to compute a system that is state-output equivalent to the hybrid system flowing according to (26) and jumping according to (28) by isolating the component of $\varsigma_{n o}$ that is also invisible during jumps. To this purpose, let $\Upsilon \in \mathbb{R}^{s_{n o}} \times \mathbb{R}^{s_{n o}}$ be the change of variable that decomposes the pair $\left(J_{n o}, J_{\text {ono }}\right)$ into the observable and unobservable parts. Namely,

$$
\Upsilon J_{n o} \Upsilon^{-1}=\left(\begin{array}{cc}
J_{n o}^{\prime} & 0 \\
\star & \star
\end{array}\right), \quad J_{\text {ono }} \Upsilon^{-1}=\left(\begin{array}{ll}
J_{\text {ono }}^{\prime} & 0
\end{array}\right),
$$

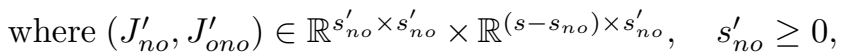
is an observable pair, with $\star$ denoting generic blocks of no interest in the subsequent developments. By changing coordinates as $\varsigma_{n o} \mapsto \varsigma_{n o}^{\prime}=\Upsilon_{\varsigma_{n o}}$ and by partitioning $\varsigma_{\text {no }}^{\prime}=\operatorname{col}\left(\varsigma_{\text {noo }}^{\prime}, \varsigma_{\text {nono }}^{\prime}\right)$ with $\varsigma_{\text {noo }}^{\prime} \in \mathbb{R}^{s_{\text {no }}^{\prime},} \varsigma_{\text {nono }}^{\prime} \in$ $\mathbb{R}^{s_{n o}-s_{n o}^{\prime}}$, it turns out that the dynamics of $\varsigma_{o}$ and $\varsigma_{n o}^{\prime}$ are described by the flow dynamics $\dot{\zeta}_{\mathrm{o}}=0, \dot{\zeta}_{\text {no }}^{\prime}=0$, and by the jump relation $\varsigma_{o}^{+}=J_{o} \varsigma_{o}+J_{\text {ono }}^{\prime} \varsigma_{\text {no }}^{\prime}, \varsigma_{\text {noo }}^{\prime+}=$ $J_{\text {no }}^{\prime} \varsigma_{\text {noo }}^{\prime}+J_{\text {noo }}^{\prime} \varsigma_{o}, \varsigma_{\text {nono }}^{\prime+}=\star$, where $J_{\text {noo }}^{\prime} \in \mathbb{R}^{s_{n o}^{\prime} \times s_{o}}$ is the matrix obtained by extracting the first $s_{n o}^{\prime}$ rows from the matrix $\Upsilon J_{\text {noo }}$, and where $\star$ denotes a linear combination of the state variables that is of no interest in the following. By keeping in mind that the output $y_{w}$ is only affected by the $\varsigma_{\mathrm{o}}$ component, it is immediately seen that $\varsigma_{\text {nono }}^{\prime}$ has no effect on the output, neither during flows nor during jumps. Hence, we conclude that system (24), (28) is state-output equivalent to the hybrid system

$$
\begin{aligned}
\dot{z}_{o} & =0, \quad \dot{z}_{n o}=0 \\
z_{o}^{+} & =N_{o} z_{o}+N_{\text {ono }} z_{n o}, z_{n o}^{+}=N_{n o} z_{n o}+N_{n o o} z_{o} \\
y_{z} & =R_{o}(\tau) z_{o}
\end{aligned}
$$

where, for notational convenience, we have re-labeled the state and the matrices as $z_{o}=\varsigma_{o} \in \mathbb{R}^{s_{o}}, z_{n o}=$ 
$\varsigma_{n o}^{\prime} \in \mathbb{R}^{s_{n o}^{\prime}}, N_{o}=J_{o}, N_{\text {ono }}=J_{\text {ono }}^{\prime}, N_{n o}=J_{n o}^{\prime}, N_{\text {noo }}=$ $J_{\text {noo }}^{\prime}$. All the state components of the previous system are visible, as is shown in the next section, where we present an asymptotic hybrid observer for this system.

\subsection{Design of the internal model}

The goal of this section is to present a methodology for the design of an internal model that has the output reproducer capabilities required in Theorem 1. In doing so, we construct a hybrid asymptotic observer for the dynamics of (29). Of the different techniques that could be used to design observers for (29), we present two methods in the following. The first is mainly inspired by techniques typically used in the context of finite-time observers and the second is motivated by high-gain observer design methodologies.

In both methods, the design of the observer for the $z_{n o}$ part (which is invisible during flows but which shows up during jumps) follows the intuition that a discretetime observer could be designed using the "measure" $z_{o}^{+}-N_{o} z_{o}$ to construct an innovation term. As $z_{o}^{+}$is not measurable we "inject" it in the $z_{n o}$ jump dynamics through the change of variables $z_{n o} \mapsto \xi_{n o}=z_{n o}+K_{2} z_{o}$ with $K_{2}$ to be fixed. By also letting $\xi_{o}=z_{o}$, in the new coordinate system (29) reads as

$$
\begin{aligned}
\dot{\xi}_{o} & =0, \quad \dot{\xi}_{n o}=0 \\
\xi_{o}^{+} & =\bar{N}_{o} \xi_{o}+\bar{N}_{o n o} \xi_{n o} \\
\xi_{n o}^{+} & =\bar{N}_{n o o} \xi_{o}+\left(N_{n o}+K_{2} N_{o n o}\right) \xi_{n o} \\
y_{\xi} & =R_{o}(\tau) \xi_{o}
\end{aligned}
$$

where $\bar{N}_{\text {ono }}:=N_{\text {ono }}, \bar{N}_{o}:=\left(N_{o}-N_{\text {ono }} K_{2}\right)$, and $\bar{N}_{\text {noo }}:=$ $N_{\text {noo }}-N_{\text {no }} K_{2}+K_{2} N_{o}-K_{2} N_{\text {ono }} K_{2}$. Using the fact that the pair $\left(N_{n o}, N_{\text {ono }}\right)$ is observable we now choose $K_{2}$ so that $\operatorname{eig}\left(N_{\text {no }}+K_{2} N_{\text {ono }}\right) \in \mathcal{D}_{1}$. We now develop two different observers for (30) according to the properties fulfilled by the pair $\left(0, R_{o}(\tau)\right)$.

\subsubsection{The pair $\left(0, R_{o}(\tau)\right)$ is not uniformly observable.}

As remarked in Section 3.1, there is no guarantee that the "visible" pair $\left(0, R_{o}(\tau)\right)$ is uniformly observable. The only guarantee is that the Gramian (27) is not singular. In such a case the asymptotic properties of the observer are mainly obtained through jumps, by integrating the output $y_{w}$ in the interval $\left[0, \tau_{\max }\right]$ to compute a "finitetime" estimate of $\xi_{o}$ (by inversion of the Gramian $\mathcal{G}_{o}$ ). Specifically, the following proposition holds.

Proposition 3 Let $\nu=2 s_{o}+s_{n o}^{\prime}, F_{\mathrm{im}}(\tau) \equiv 0_{\nu \times \nu}$,

$$
G_{\mathrm{im}}(\tau)=\left(\begin{array}{c}
R_{o}^{T}(\tau) \\
0_{\nu-s_{o} \times m}
\end{array}\right)
$$

$$
\Sigma_{\mathrm{im}}=\left(\begin{array}{ccc}
0_{s_{o} \times s_{o}} & 0_{s_{o} \times s_{o}} & 0_{s_{o} \times s_{n o}^{\prime}} \\
\bar{N}_{o} \mathcal{G}_{o}^{-1} & 0_{s_{o} \times s_{o}} & \bar{N}_{\text {ono }} \\
\bar{N}_{\text {noo }} \mathcal{G}_{o}^{-1} & 0_{s_{n o}^{\prime} \times s_{o}} & N_{\text {no }}+K_{2} N_{\text {ono }}
\end{array}\right)
$$

and $\Gamma_{\mathrm{im}}(\tau)=\left(0_{m \times s_{o}} R_{o}(\tau) 0_{m \times s_{n o}^{\prime}}\right)$. Then the quadruplet $\left(F_{\mathrm{im}}, G_{\mathrm{im}}(\cdot), \Gamma_{\mathrm{im}}(\cdot), \Sigma_{\mathrm{im}}\right)$ has the hybrid internal model property.

The proof of this proposition is presented in Appendix $\mathrm{B}$. In the structure of the observer it is possible to isolate a state variable $\eta_{i}$, with flow dynamics given by $\dot{\eta}_{i}=$ $R_{o}^{T}(\tau) y_{\xi}$ and jump map $\eta_{i}^{+}=0$, whose goal is to estimate the state component $\xi_{o}$ of $(30)$ at the beginning $\left(\xi_{o}(0)\right)$ and at the end $\left(\xi_{o}\left(\tau_{\max }\right)\right)$ of the time interval through the relation (B.1) and to use those estimates at the jump time to enforce a cascade structure of the error system (see the proof of the proposition).

\subsubsection{The pair $\left(0, R_{o}(\tau)\right)$ is uniformly observable.}

An alternative observer design can be proposed if the visible pair $\left(0, R_{o}(\tau)\right)$ is also uniformly observable in the interval in the sense specified below. In such a case the asymptotic properties of the observer are mainly obtained through flows, by designing a high-gain observer with a fast estimation rate. In the following the uniform observability condition is considered "output-wise" as formalized in the next assumption.

Assumption 5 Let $R_{o}(\tau)=\operatorname{col}\left(R_{o 1}(\tau), \ldots R_{o m}(\tau)\right)$. Then for all $i=1, \ldots, m$ the observability matrices

$$
\mathcal{O}_{i}(\tau)=\operatorname{col}\left(R_{o i}(\tau) \dot{R}_{o i}(\tau) \cdots R_{o i}^{\left(s_{o}-1\right)}(\tau)\right)
$$

are non-singular for all $\tau \in\left[0, \tau_{\max }\right]$.

Under this assumption, the observer for the system (30) can be constructed by using high-gain tools to estimate, during flows, the observable component of the system. Instrumental to the design of the observer is the transformation of system (30) into a canonical observability form following the arguments in Bestle \& Zeitz (1983).

Let $P_{i}(\tau):\left[0, \tau_{\max }\right] \rightarrow \mathbb{R}^{s_{o} \times s_{o}}, i=1, \ldots, m$, be defined as (see Bestle \& Zeitz (1983))

$$
P_{i}(\tau)^{-1}=\left[q_{i}(\tau) \tilde{\mathcal{L}} q_{i}(\tau) \ldots \tilde{\mathcal{L}}^{s_{o}-1} q_{i}(\tau)\right]
$$

where $q_{i}(\tau)$ is the last column of $\mathcal{O}_{i}(\tau)^{-1}$ and $\tilde{\mathcal{L}}(\cdot)$ is the differential operator $\tilde{\mathcal{L}} q_{i}(\tau):=-\dot{q}_{i}(\tau)$. By defining $\chi_{o} \in \mathbb{R}^{m s_{o}}$ and $\chi_{n o}$ as $\chi_{o}=\mathbf{P}(\tau) \xi_{o}$ and $\chi_{n o}=\xi_{n o}$ with $\mathbf{P}(\tau)=\operatorname{col}\left(P_{1}(\tau), \cdots, P_{m}(\tau)\right)$, the arguments in Bestle $\&$ Zeitz (1983) show that the flow dynamics of $\chi_{o}$ and $\chi_{n o}$ are described by

$$
\dot{\chi}_{o}=(\mathbf{A}+\mathbf{r}(\tau) \mathbf{C}) \chi_{o}, \quad \dot{\chi}_{n o}=0
$$


where $\mathbf{A}=\operatorname{blkdiag}(A, \ldots, A), \mathbf{C}=\operatorname{blkdiag}(C, \ldots, C)$, $\mathbf{r}(\tau)=\operatorname{blkdiag}\left(r_{1}(\tau), \ldots, r_{m}(\tau)\right)$, with

$$
A=\left(\begin{array}{cc}
0_{1 \times s_{o}-1} & 0 \\
I_{s_{o}-1} & 0_{s_{o}-1 \times 1}
\end{array}\right), \quad C=\left(\begin{array}{ll}
0_{1 \times s_{o}-1} & 1
\end{array}\right)
$$

and with $r_{i}:\left[0, \tau_{\max }\right] \rightarrow \mathbb{R}^{s_{o} \times 1}, i=1, \ldots, m$, appropriately defined smooth functions. Furthermore, $y_{\xi}=\mathbf{C} \chi_{o}$. Finally, the jump relations of $\chi_{o}$ and $\chi_{n o}$ can be easily computed as

$$
\begin{aligned}
\chi_{o}^{+} & =\mathbf{M}_{o} \chi_{o}+\mathbf{M}_{\text {ono }} \chi_{n o} \\
\chi_{n o}^{+} & =\left(N_{n o}+K_{2} N_{\text {ono }}\right) \chi_{n o}+\mathbf{M}_{n o o} \chi_{o}
\end{aligned}
$$

where $\mathbf{M}_{o}=\operatorname{blkdiag}\left(M_{o 1}, \ldots, M_{\text {om }}\right), \mathbf{M}_{\text {ono }}=\operatorname{col}\left(M_{\text {ono } 1}\right.$, $\left.\ldots, M_{\text {ono } m}\right), \mathbf{M}_{\text {noo }}=\left(M_{\text {noo } 1}, \ldots, M_{\text {noo } m}\right)$, with $M_{\text {ono } i}=P_{i}(0) \bar{N}_{\text {ono }}, M_{\text {o } i}=P_{i}(0) \bar{N}_{o} P_{i}\left(\tau_{\max }\right)^{-1}$, $M_{\text {noo } i}=\bar{N}_{\text {noo }} P_{i}\left(\tau_{\max }\right)^{-1}, i=1, \ldots, m$. The following proposition, proved in Appendix $\mathrm{C}$, shows that an asymptotic observer for the $\chi_{o}$ and $\chi_{n o}$ dynamics can always be designed.

Proposition 4 Let $\mathbf{K}_{1}=\operatorname{blkdiag}\left(K_{1}, \ldots K_{1}\right)$ with

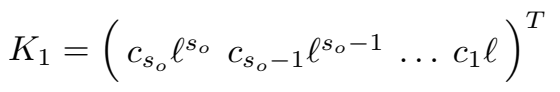

where the $c_{i}$ 's are coefficients of a Hurwitz polynomial and $\ell$ is a design parameter. Let $\nu=m s_{o}+s_{n o}^{\prime}$,

$$
\begin{aligned}
& F_{\mathrm{im}}=\left(\begin{array}{cc}
\mathbf{A}+\mathbf{K}_{1} \mathbf{C} & 0_{m s_{o} \times s_{n o}^{\prime}} \\
0_{s_{n o}^{\prime} \times m s_{0}} & 0_{s_{n o}^{\prime} \times s_{n o}^{\prime}}
\end{array}\right), G_{\mathrm{im}}(\tau)=\left(\begin{array}{c}
\mathbf{r}(\tau)-\mathbf{K} \\
0_{s_{n o}^{\prime} \times m}
\end{array}\right), \\
& \Sigma_{\mathrm{im}}=\left(\begin{array}{cc}
\mathbf{M}_{o} & \mathbf{M}_{\text {ono }} \\
\mathbf{M}_{\text {noo }} & N_{n o}+K_{2} N_{\text {ono }}
\end{array}\right),
\end{aligned}
$$

and $\Gamma_{\mathrm{im}}=\left(\mathbf{C}, 0_{m \times s_{n o}^{\prime}}\right)$. Then there exists a $\ell^{\star} \geq 1$ such that for all $\ell \geq \ell^{\star}$ the quadruplet $\left(F_{\mathrm{im}}, G_{\mathrm{im}}(\tau), \Sigma_{\mathrm{im}}, \Gamma_{\mathrm{im}}\right)$ has the hybrid internal model property.

\section{Splines as Hybrid Exosystems}

Spline interpolation is widely adopted in the robotic literature (Siciliano et al. (2009)) in order to generate reference signals that smoothly interpolate way-points by avoiding Runge's phenomenon, which usually appears while using polynomial interpolation. Splines have been shown to be effective in path generation for mobile robots Howard \& Kelly (2006), in the aerospace domain Sonneveldt et al. (2009), and in many other applicative fields where efficient trajectory planning is a key requirement.

We are interested in cyclic time signals $y^{\star}(t)$ obtained by periodically concatenating the basic signal, $\mathcal{B}(t)$, with a period given by $T$, where

$$
y^{\star}(t)=\mathcal{B}(t-i T), \quad i=\left\lfloor\frac{t}{T}\right\rfloor, t \geq 0, T>0,
$$

with $\mathcal{B}:[0, T] \rightarrow \mathbb{R}$ generated by using splines that pass through $N$ points $\left\{p_{1}, p_{2}, \ldots, p_{N}\right\}$ at time instances $\left\{t_{1}, t_{2}, \ldots, t_{N}\right\}$, namely $\mathcal{B}\left(t_{k}\right)=p_{k}, k=1, \ldots, N$. It is assumed that $t_{1}=0, t_{N}=T-T / N$ and $t_{k+1}-t_{k}=T / N$ for all $k=1, \ldots, N-1$. We also assume that $\mathcal{B}(\cdot)$ is such that $\mathcal{B}(0)=\mathcal{B}(T)=p_{1}$ so that $y^{\star}: \mathbb{R}_{\geq 0} \rightarrow \mathbb{R}$ is a continuous function (see Figure 1).

The idea behind spline generation (see Schumaker

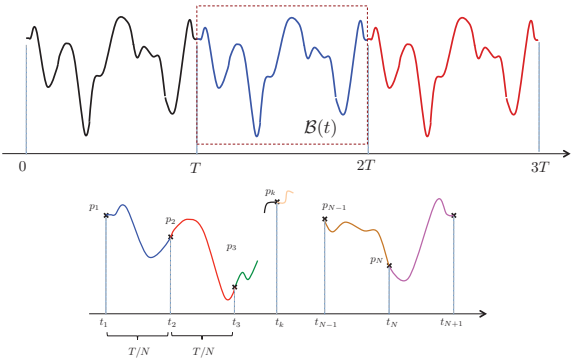

Fig. 1. Periodically concatenated splines.

$(2007))$ is to interpolate $N$ polynomials $\mathcal{P}_{k}(t)$ : $\left[t_{k}, t_{k+1}\right] \rightarrow \mathbb{R}$ of suitable order to guarantee that $\mathcal{P}_{k}\left(t_{k}\right)=p_{k}, \mathcal{P}_{k}\left(t_{k+1}\right)=p_{k+1}, k=1, \ldots, N$ (with $t_{N+1}=T$ and $p_{N+1}=p_{1}$ ) and to smooth the time derivatives of the signal $y^{\star}(t)$ at the times $t_{k}$, $k=1, \ldots, N+1$. For instance, by using polynomials of order three, simple algebraic arguments can be used to show that it is possible to design the four coefficients of the $N$ polynomials in such a way that the first and second time derivative (velocities and accelerations) of $y^{\star}(t)$ are continuous at $t=t_{k}, k=2, \ldots, N+1$. Smoother signals can be obtained by increasing the order of the polynomials $\mathcal{P}_{k}$. From now on, the polynomials $\mathcal{P}_{k}$ are assumed to be fixed in order to have continuity of $y^{\star}(t), \dot{y}^{\star}(t)$ and $\ddot{y}^{\star}(t)$ for all $t \geq 0$.

Next, we are interested in computing $y^{\star}(t)$ as the output of a hybrid linear exosystem of the form

$$
\begin{aligned}
& \dot{\tau}=1, \quad \dot{w}=S w, \quad(\tau, w) \in\left[0, \tau_{\max }\right] \times \mathbb{R}^{s} \\
& \tau^{+}=0, \quad w^{+}=J w, \quad(\tau, w) \in\left\{\tau_{\max }\right\} \times \mathbb{R}^{s} \\
& y^{\star}=Q w
\end{aligned}
$$

where $Q=\left[\begin{array}{ll}Q_{1} & 0_{1 \times N}\end{array}\right]$, the matrices $S$ and $J$ are to be defined, and $\tau_{\max }:=T / N$. The clock variable $\tau$ determines the polynomial switching times. The construction of the exosystem is aided by breaking it into two substates, $w_{1} \in \mathbb{R}^{4}, w_{2} \in \mathbb{R}^{N}$, with the dimension of the sub-state $w_{1}$ dependent on the order of the polynomials 
that are used as the basic spline functions.

Let $S_{1} \in \mathbb{R}^{4} \times \mathbb{R}^{4}$ be the matrix whose elements are all zero except along the super-diagonal, which is filled with ones, and let $Q_{1}=\left(\begin{array}{ll}1 & 0_{1 \times 3}\end{array}\right)$. Furthermore, let

$$
\mathcal{Q}:=\left(\begin{array}{c}
Q_{1} \\
Q_{1} S_{1} \\
Q_{1} e^{S_{1} \tau_{\max }} \\
Q_{1} S_{1} e^{S_{1} \tau_{\max }}
\end{array}\right), \mathbf{v}_{k}:=\left(\begin{array}{c}
p_{k} \\
v_{k} \\
p_{k+1} \\
v_{k+1}
\end{array}\right), k=1, \ldots, N
$$

with $v_{k}=\lim _{t \rightarrow t_{k}^{+}} \dot{\mathcal{P}}_{k}(t)$ and $v_{k+1}=\lim _{t \rightarrow t_{k+1}^{-}} \dot{\mathcal{P}}_{k}(t)$. The initial condition of the component $w_{1}$ of the exosystem is set to $w_{1}\left(t_{1}\right)=\mathcal{Q}^{-1} \mathbf{v}_{1}$, so that $y^{\star}(t)=Q_{1} w_{1}(t)$ for $t \in\left[t_{1}, t_{2}\right]$.

Then we have to identify the switching rule of the state of the exosystem at times $t_{k}, k=2, \ldots, N+1$ in order to reproduce the reference at times $t>t_{2}$. To this purpose, we observe that the value $w_{1}^{+}\left(t_{k+1}\right)$ needed to reproduce $\mathcal{P}_{k+1}(t)$ with $t \in\left[t_{k+1}, t_{k+2}\right], k=1, \ldots, N-1$, is

$$
w_{1}^{+}\left(t_{k+1}\right)=\mathcal{Q}^{-1} \mathbf{v}_{k+1} .
$$

We observe that $p_{k+1}$ and $v_{k+1}$ can be expressed as functions of $w_{1}\left(t_{k+1}\right)$ since $p_{k+1}=Q_{1} w_{1}\left(t_{k+1}\right)$, and $v_{k+1}=Q_{1} S_{1} w_{1}\left(t_{k+1}\right)$. It is possible to express $v_{k+2}$ as a function of $p_{k}, p_{k+1}, p_{k+2}, v_{k}$ and $v_{k+1}$ by imposing continuity in the acceleration at time $t_{k+1}$. In fact, by imposing $Q_{1} S_{1}^{2} w_{1}\left(t_{k+1}\right)=Q_{1} S_{1}^{2} w_{1}\left(t_{k+1}\right)^{+}$, and using

$$
w_{1}\left(t_{k+1}\right)=e^{S_{1} \tau_{\max }} w_{1}\left(t_{k}\right)^{+}=e^{S_{1} \tau_{\max }} \mathcal{Q}^{-1} \mathbf{v}_{k}
$$

one obtains $Q_{1} S_{1}^{2} e^{S_{1} \tau_{\max }} \mathcal{Q}^{-1} \mathbf{v}_{k}=Q_{1} S_{1}^{2} \mathcal{Q}^{-1} \mathbf{v}_{k+1}$, which, solved for $v_{k+2}$, yields

$$
v_{k+2}=\Gamma\left(\begin{array}{lllll}
p_{k} & p_{k+1} & p_{k+2} & v_{k} & v_{k+1}
\end{array}\right)^{T}
$$

with $\Gamma=\left(-3 / \tau_{\max } 03 / \tau_{\max }-1-4\right)$. By embedding the previous relation in (34) we obtain

$$
w_{1}^{+}\left(t_{k+1}\right)=L\left(\begin{array}{lllll}
p_{k} & p_{k+1} & p_{k+2} & v_{k} & v_{k+1}
\end{array}\right)^{T},
$$

with $L \in \mathbb{R}^{4 \times 5}$ properly defined. Now, we observe that $p_{k}=Q_{1} e^{-S_{1} \tau_{\max }} w_{1}\left(t_{k+1}\right), p_{k+1}=Q_{1} w_{1}\left(t_{k+1}\right), v_{k}=$ $Q_{1} S_{1} e^{-S_{1} \tau_{\max }} w_{1}\left(t_{k+1}\right), v_{k+1}=Q_{1} S_{1} w_{1}\left(t_{k+1}\right)$. To write a relation of the form $w^{+}\left(t_{k+1}\right)=J w\left(t_{k+1}\right)$ we are thus left to express $p_{k+2}$ as a function of the state of the exosystem. In order to preserve the linearity of the exosystem, this can be done by "enriching" the exosystem with additional states $w_{2} \in \mathbb{R}^{N}$ governed by the following dy- namics (implementing a shift register)

$\dot{w}_{2}=0, w_{2}^{+}=J_{22} w_{2}$, where $J_{22}=\left(\begin{array}{cc}0_{1 \times N-1} & 1 \\ I_{N-1} & 0_{N-1 \times 1}\end{array}\right)$,

with the initial condition $w_{2}\left(t_{1}\right)=\left(\begin{array}{ll}1 & 0_{1 \times N-1}\end{array}\right)^{T}$. In this way $p_{k+2}=P w_{2}\left(t_{k+1}\right)$, with $P=\left(\begin{array}{llll}p_{3} \ldots p_{N} & p_{1} p_{2}\end{array}\right)$, and thus the exosystem (33) takes the form

$$
\begin{aligned}
& \left.\dot{\tau}=1, \dot{w}_{1}=S_{1} w_{1}, \dot{w}_{2}=0\right\}(\tau, w) \in\left[0, \tau_{\max }\right] \times \mathbb{R}^{s} \\
& \left.\begin{array}{rl}
\tau^{+} & =0, w_{1}^{+}=J_{11} w_{1}+J_{12} w_{2} \\
w_{2}^{+} & =J_{22} w_{2}
\end{array}\right\}(\tau, w) \in\left\{\tau_{\max }\right\} \times \mathbb{R}^{s}
\end{aligned}
$$

with $y^{\star}=Q_{1} w_{1}$, where

$$
S_{1}=\left(\begin{array}{cc}
0_{3 \times 1} & I_{3} \\
0_{1 \times 4}
\end{array}\right), J_{11}=\left(\begin{array}{cc}
I_{3} & 0_{3 \times 1} \\
& L_{1}
\end{array}\right), J_{12}=\left(\begin{array}{c}
0_{3 \times N} \\
L_{2}
\end{array}\right),
$$

with $L_{1}$ and $L_{2}$ row vectors appropriately defined. The matrices $S$ and $J$ are then implicitly defined.

It is interesting to note that, due to the specific structure of $S, J$ and $Q$, system (33) is neither dynamically observable through the flow dynamics nor statically observable through the jump relation, namely the pairs $(S, Q)$ and $(J, Q)$ are not detectable. In particular, due to the structure of $S$ and $Q$, it is apparent that the $w_{2}$ component is not detectable through the output $Q w$ of the continuous-time system $\dot{w}=S w$. Similarly, the structures of $J$ and $Q$ imply that $(Q w)^{+}=Q w$, namely the state $w$, and thus, in particular, $w_{2}$, does not show up on the output during jumps. Nevertheless, denoting by $w_{14}$ the fourth component of $w_{1}$ and noting that the jump rule governing $w_{14}$ is given by $w_{14}^{+}=L_{1} w_{1}+L_{2} w_{2}$, it turns out that $w_{2}$ shows up during jumps by affecting the value of $w_{14}$ that, in turn, is dynamically observable by the output $Q_{1} w_{1}$ of the flow dynamics $\dot{w}_{1}=S_{1} w_{1}$.

\section{Example: High-Precision Robust Tracking of a Spline Reference Signal by a UAV}

We consider the problem of high-precision tracking of spline-generated trajectories in the lateral and longitudinal position of a rotary wing UAV such as a quadrotor. The forthcoming developments can be easily generalized to other kinds of under-actuated vehicles, such as ducted-fans, helicopters, coaxials, and multi-rotors.

Denoting by $(x, y, z)$ the lateral, longitudinal and vertical position of the UAV expressed with respect to an inertial reference frame, and by $(\theta, \phi, \psi)$ the roll, pitch 
and yaw angles, it turns out that

$$
M\left(\begin{array}{c}
\ddot{x} \\
\ddot{y} \\
\ddot{z}
\end{array}\right)=R(\theta, \phi, \psi)\left(\begin{array}{c}
0 \\
0 \\
-K_{T}
\end{array}\right) \omega_{e}^{2}+\left(\begin{array}{c}
0 \\
0 \\
M \mathrm{~g}
\end{array}\right),
$$

where $R(\theta, \phi, \psi)$ is the rotation matrix parametrized in term of roll, pitch and yaw angles, $M$ is the mass of the vehicle, $\mathrm{g}$ is the gravitational acceleration, $K_{T}$ is the thrust coefficient (assumed equal for all the rotors), and $\omega_{e}^{2}$ is the sum of the squares of the rotational speeds of the four propellers. In the previous model $K_{T}$ is regarded as an uncertain parameter.

For the sake of simplicity we do not take into account all of the kinematics and dynamics of the vehicle by implicitly considering the roll and the pitch angles of the vehicle as virtual inputs for the lateral and longitudinal dynamics ${ }^{3}$. Using the fact that the element of $R$ in the third row and third column is equal to $C_{\theta} C_{\phi}$, we start by feedback linearizing the vertical dynamics by choosing a proportional integral controller of the propeller speed,

$$
\omega_{e}^{2}=\frac{1}{K_{T}^{o} C_{\theta} C_{\phi}}\left(v_{1}+M \mathrm{~g}+\xi\right), \quad \dot{\xi}=v_{2}
$$

in which $K_{T}^{o}$ is a nominal value of $K_{T}$, and $v_{1}$ and $v_{2}$ are two residual inputs to be chosen in order to stabilize the system $M \ddot{z}=-\mu\left(v_{1}+M \mathrm{~g}+\xi\right)+M \mathrm{~g}, \dot{\xi}=v_{2}$ with $\mu=K_{T} / K_{T}^{o}$ an uncertain parameter. A dynamic robust controller processing the available measures $(z, \xi)$ can be used for this purpose. Standard linear arguments then show that the resulting closed-loop vertical system is such that $z \rightarrow 0$ no matter the actual value of $\mu$.

As a consequence of this choice, the lateral and longitudinal dynamics read as

$$
M\left(\begin{array}{l}
\ddot{x} \\
\ddot{y}
\end{array}\right)=\mu R(\psi) V(\theta, \phi)\left(v_{1}+M g+\xi\right)
$$

where $R(\psi)$ is the $2 \times 2$ elementary rotation matrix whose first and second row are respectively $\left(C_{\psi}-S_{\psi}\right),\left(S_{\psi} C_{\psi}\right)$, and $V(\theta, \phi)=\operatorname{col}\left(\tan \theta,-\frac{\tan \phi}{C_{\theta}}\right)$. Note that $V(\theta, \phi)$ is invertible for all $\theta, \phi \in(-\pi / 2, \pi / 2)$. We thus design the two (virtual) inputs $(\theta, \phi)$ so that

$$
V(\theta, \phi)=\frac{1}{v_{1}+M \mathrm{~g}+\xi} R(\psi)^{T} u
$$

\footnotetext{
3 By following standard nomenclature in the literature of control of under-actuated vehicles, we just consider the design of the "outer loop". Standard "backstepping" tools can then be adopted to obtain the true torque input starting from a virtual control law for the roll and pitch angles.
}

where $u=\left(u_{x}, u_{y}\right) \in \mathbb{R}^{2}$ is a residual input, so as to obtain $M \operatorname{col}(\ddot{x}, \ddot{y})=\mu u$, namely two double integrators independently controlled by the inputs $u_{x}$ and $u_{y}$. This system is clearly in the Brunowsky form (5) with $\bar{B}=\frac{\mu}{M} I_{2}$ and with all the other matrices equal to zero. In particular the system has vector relative degree $(2,2)$. The goal for this system is to robustly track a spline trajectory which passes through the points $\{(1,1),(1,2),(1,3),(2,3),(2,2),(2,1)\}$ in a cycle (see Figure 2). The spline in the plane can be obtained by generating spline trajectories of the form indicated in the previous section for each of the $x$ and $y$ coordinates. According to the theory in Section 4, each spline can be thought of as being generated by an exosystem of dimension 10 (as $N=6$ ). In particular, the reference signals for the $x$ and $y$ coordinates can be thought of as being generated by $\dot{w}_{x}=S_{x} w_{x}, y_{x}^{\star}=Q_{x} w_{x}$ and $\dot{w}_{y}=S_{y} w_{y}, y_{y}^{\star}=Q_{y} w_{y}$ where $Q_{x} \in \mathbb{R}^{1 \times 10}, Q_{y} \in \mathbb{R}^{1 \times 10}$, $S_{x} \in \mathbb{R}^{10 \times 10}$ and $S_{y} \in \mathbb{R}^{10 \times 10}$ are constructed as in Section 4 . The overall exosystem thus has dimension $s=20$ with $S=\operatorname{blkdiag}\left(S_{x}, S_{y}\right)$ and the $Q$ in (5) having the form $Q=\operatorname{blkdiag}\left(Q_{x}, Q_{y}\right)$. In this case the hybrid regulator equation can be easily solved yielding an expression for $R(\tau)$ of the form $R(\tau)=\bar{B}^{-1} Q S^{2}$. Note that $R(\cdot)$ is affected by an uncertain parameter $\mu$ entering in $\bar{B}$. By bearing in mind Proposition 1, the fact that just one uncertain parameter affects $\bar{B}$, and thus $R(\cdot)$, in a scalar multiplicative way, implies that no duplication of $R(\tau)$ is needed for robust design, namely $\mathbf{R}(\tau)=R(\tau)$ and $\mathbf{s}=s=20$.

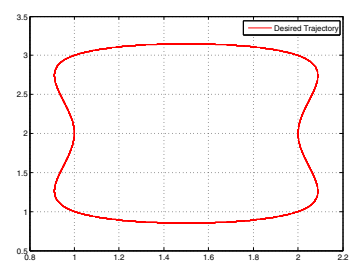

Fig. 2. 2-D Quadrotor Trajectory

It turns out that for the spline tracking problem, the procedure for designing the quadruplet $\left(F_{\mathrm{im}}, G_{\mathrm{im}}, \Gamma_{\mathrm{im}}(\tau), \Sigma_{\mathrm{im}}\right)$ presented in Proposition 2 fails because it produces no guarantee that the resultant $\Gamma_{\mathrm{im}}(\tau)$ is continuous. The details of the failure of this approach are presented by Cox et al. (2012).

Motivated by the failure of Proposition 2, we implement the design procedure presented in Section 3. Specifically, we follow the design procedure presented in Proposition 3 applied separately for the two double integrators. We define the errors $e_{x}=x-Q_{x} w_{x}$ and $e_{y}=y-Q_{y} w_{y}$ as the only available measures and utilize the high-gain observer presented in Theorem 1 (see also Cox et al. (2014)) to reconstruct velocities. Since the vector relative degree is $(2,2)$, the high-gain observer state $\chi$ is in $\mathbb{R}^{4}$ (dimension 2 for each double integrator) and can be 
partitioned coherently with the notation used in Section 2.3 as $\chi=\operatorname{col}\left(\chi_{x}, \chi_{y}\right)$.

By following the theory in Section 3.1, it turns out that the Gramian $\mathcal{G}$ in this specific case has rank 16, meaning that the dimension of the states that are visible during flows is $s_{o}=4$, two for each input channel. As for the dimension of the dynamics that are invisible during flows but that become visible during jumps, it turns out that $s_{n o}^{\prime}=9$, specifically 5 for the $x$-channel and 4 for the $y$-channel. Considering the additional state $\eta_{i}$ of Proposition 3 has dimension $s_{o}$, it follows that the full internal model $\left(\eta_{i}, \eta_{o}, \eta_{n o}\right)$ has dimension 17 , namely 9 for the $x$-channel and 8 for the $y$-channel.

For the choice of $K_{2}$ we solved an LQR problem using identity matrices for the weighting parameters. For the stabilizer feedback we choose $K=\operatorname{blkdiag}\left(K_{x}, K_{y}\right)$ with both $K_{x}$ and $K_{y}$ taken as (11) and with the highgain parameter $\kappa=10^{2}$.

The observer for the plant state is designed with the choice $\Lambda_{\text {st }_{i}}=\operatorname{col}\left(2 g, g^{2}\right)$, the high-gain parameter $g=10^{2}$, from which $\Lambda_{\mathrm{st}}$ and $\Phi_{\mathrm{st}}$ are defined via Theorem 1. Recall from the discussion of the regulator design just above Theorem 1 that $\Lambda_{\text {st }}$ and $\Phi_{\text {st }}$ are block diagonal matrices with $\Lambda_{\mathrm{st}_{i}}$ and $\Phi_{\mathrm{st}_{i}}$ the block elements. In this case $m=2$ so there are two block elements.

The results from these regulator choices can be seen in Figure 3. In order to test the robustness of the regulator we simulated a large change on the parameter $\mu$ at time $t=36$, with $\mu$ instantaneously switching from $\mu=0.8$ to $\mu=0.3$. From a physical view point, the decrease in the thrust coefficient can be motivated by battery discharge. Due to the high-gain feedback inherent in the stabilizer unit used for the regulator, there are some large spikes in the input from peaking. In fact we implement a saturation for the input to avoid some of the larger peaking effects.

A further numerical analysis has been made by imple-
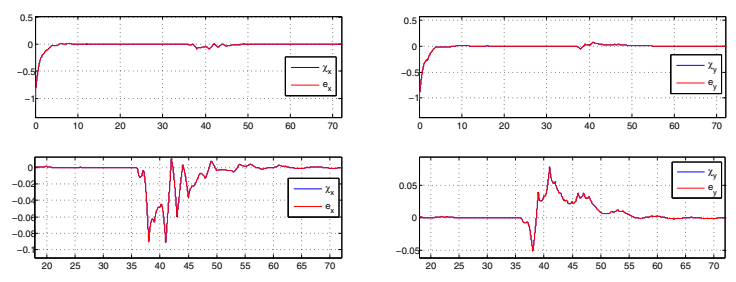

Fig. 3. Internal model-based design. Error in the $x$-coordinate; Error in the $y$-coordinate

menting a controller without an internal model, namely a controller of the form $u=R(\tau) w-\kappa \bar{B}^{-1} K \chi$, which uses a nominal feedforward term (the $R(\tau)$ in the regulator has been taken with $\mu=1$ ) to nominally track the desired trajectory, along with the stabilization feedback (with state $\chi$ as above). The results are shown in Figures 4 in which the large tracking error can be observed.
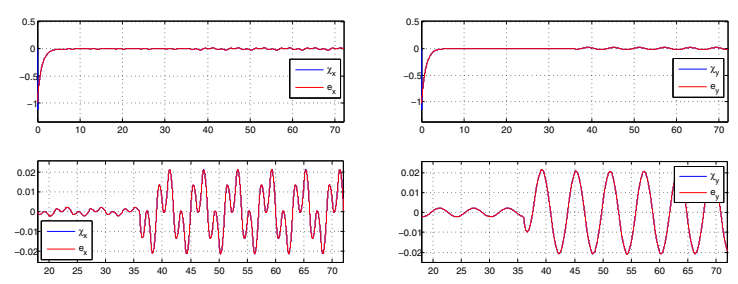

Fig. 4. Regulator without internal model. Error in the $x$-coordinate; Error in the $y$-coordinate

\section{Conclusions}

In the paper we tackled the problem of hybrid output regulation for a class of linear hybrid systems and exosystems with periodic jump times. The procedure to design robust regulators relies on a notion of "visibility" of the so-called hybrid steady-state generator system. The internal model has been designed by using tools and ideas that are typical in the design of finite-time and highgain observers, specialized to the specific hybrid context. The developed theory has then been specialized to the case in which the hybrid exosystem is designed to reproduce spline trajectories. Future works on the subject are mainly directed to relax the assumption of a fixed dwell time in the clock dynamics and to extend the theory developed so far to the case of nonlinear hybrid plants and exosystems.

\section{References}

Bestle, D. \& Zeitz, M. (1983), 'Canonical form observer design for non-linear time-variable systems', International Journal of Control 38(2), 419-431.

Carnevale, D., Galeani, S. \& Menini, L. (2012a), Output regulation for a class of linear hybrid systems. part 1: trajectory generation, in 'Decision and Control (CDC), 2012 IEEE 51st Annual Conference on', pp. 6151-6156.

Carnevale, D., Galeani, S. \& Menini, L. (2012b), Output regulation for a class of linear hybrid systems. part 2: stabilization, in 'Decision and Control (CDC), 2012 IEEE 51st Annual Conference on', pp. 6157-6162.

Carnevale, D., Galeani, S. \& Menini, L. (2013), A case study for hybrid regulation: Output tracking for a spinning and bouncing disk, in 'Control Automation (MED), 2013 21st Mediterranean Conference on', pp. 858-867.

Cox, N., Marconi, L. \& Teel, A. R. (2011), Hybrid output regulation with unmeasured clock, in 'Decision and Control and European Control Conference (CDCECC), 2011 50th IEEE Conference on', pp. 7410-7415.

Cox, N., Marconi, L. \& Teel, A. R. (2012), Hybrid internal models for robust spline tracking, in 'Decision and Control (CDC), 2012 IEEE 51st Annual Conference on', pp. 4877-4882.

Cox, N., Marconi, L. \& Teel, A. R. (2013), Results on non-linear hybrid output regulation, in 'Decision and 
Control (CDC), 2013 IEEE 52nd Annual Conference on', pp. 2036-2041.

Cox, N., Marconi, L. \& Teel, A. R. (2014), 'High-gain observers and linear output regulation for hybrid exosystems', International Journal of Robust and Nonlinear Control 24(6), 1043-1063.

Cox, N., Teel, A. R. \& Marconi, L. (2011), Hybrid output regulation for minimum phase linear systems, in 'American Control Conference (ACC), 2011', pp. 863868.

D'Alessandro, P., Isidori, A. \& Ruberti, A. (1973), 'A new approach to the theory of canonical decomposition of linear dynamical systems', SIAM Journal on Control 11(1), 148-158.

Francis, B. A. \& Wonham, W. M. (1976), 'The internal model principle of control theory', Automatica $12(5), 457-465$.

Francis, B. \& Wonham, W. M. (1975), 'The internal model principle for linear multivariable regulators', Applied Mathematics and Optimization 2(2), 170-194.

Goebel, R., Sanfelice, R. G. \& Teel, A. R. (2009), 'Hybrid dynamical systems', IEEE Control Systems Magazine $\mathbf{2 9}(2), 28-93$.

Goebel, R., Sanfelice, R. G. \& Teel, A. R. (2012), Hybrid Dynamical Systems: Modeling, Stability, and Robustness, Princeton University Press.

Howard, T. M. \& Kelly, A. (2006), Trajectory and spline generation for all-wheel steering mobile robots, in 'Intelligent Robots and Systems, 2006 IEEE/RSJ International Conference on', pp. 4827-4832.

Marconi, L. \& Teel, A. R. (2010), A note about hybrid linear regulation, in 'Decision and Control (CDC), 2010 49th IEEE Conference on', pp. 1540-1545.

Marconi, L. \& Teel, A. R. (2013), 'Internal model principle for linear systems with periodic state jumps', $A u$ tomatic Control, IEEE Transactions on 58(11), 27882802.

Schumaker, L. (2007), Spline Functions: Basic Theory, third edn, Cambridge Mathematical Library.

Siciliano, B., Sciavicco, L. \& Villani, L. (2009), Robotics: Modeling, Planning and Control, Springer.

Sonneveldt, L., Oort, E. R. V., Chu, Q. P. \& Mulder, J. A. (2009), Nonlinear adaptive flight control law design and handling qualities evaluation, in 'Decision and Control, 2009 held jointly with the 2009 28th Chinese Control Conference. CDC/CCC 2009. Proceedings of the 48th IEEE Conference on', pp. 7333-7338.

Stewart, G. W. (1969), 'On the continuity of the generalized inverse', SIAM Journal on Applied Mathematics $\mathbf{1 7}(1)$, pp. 33-45.

Zhang, Z. \& Serrani, A. (2006), 'The linear periodic output regulation problem', Systems and Control Letters $\mathbf{5 5}(7), 518-529$.

\section{A Proof of Theorem 1}

The closed-loop system analyzed here is the combination of the plant in Brunovsky's canonical form (5) and the exosystem (2), along with the regulator consisting of an internal model unit and a stabilization unit (23). By defining error variables as $\tilde{z}:=z-\Pi_{z}(\tau) w$, $\tilde{\xi}:=\xi-\Pi_{\xi}(\tau) w, \tilde{y}_{r}:=y_{r}-\Pi_{y_{r}}(\tau) w, \tilde{\eta}:=\eta-\Pi_{\eta}(\tau) w$ and bearing in mind the hybrid regulator equations (4) (in the forms (6)-(12)), Assumption 3, and the equations (22), it turns that the closed-loop system in the error coordinates is described by

$$
\begin{aligned}
\dot{\tau} & =1, \quad \dot{\tilde{z}}=A_{11} \tilde{z}+A_{12} L \tilde{\xi}, \quad \dot{\tilde{\xi}}=A_{22} \tilde{\xi}+A_{23} \tilde{y}_{r} \\
\dot{\tilde{y}}_{r} & =A_{31} \tilde{z}+A_{32} \tilde{\xi}+A_{33} \tilde{y}_{r}+\bar{B}(u-R(\tau) w) \\
\dot{\tilde{\eta}} & =F_{\mathrm{im}}(\tau) \tilde{\eta}+G_{\mathrm{im}}(\tau)(u-R(\tau) w) \\
\dot{\chi} & =\Phi_{\mathrm{st}} \chi+\Lambda_{\mathrm{st}} L \tilde{\xi}
\end{aligned}
$$

during flows, and $\tau^{+}=0, \tilde{z}^{+}=\tilde{z}, \tilde{\xi}^{+}=\tilde{\xi}, \tilde{y}_{r}^{+}=\tilde{y}_{r}$ $\tilde{\eta}^{+}=\Sigma_{\text {im }} \tilde{\eta}, \chi^{+}=\chi$ during jumps, where the timing of the jumps is controlled by the clock variable $\tau$, and where $R(\tau)$ is defined by (12). In these coordinates the error reads as $e=L \tilde{\xi}$.

There are three additional changes of variables that help in order to perform the stability analysis of the error system. The first aims to deal with the relative degree of the system by re-defining the variable $\tilde{y}_{r}$ as $\tilde{y}_{r} \rightarrow \tilde{e}_{r}=\tilde{y}_{r}+\bar{H} \tilde{\xi}$, where the matrix $\bar{H}=\operatorname{blkdiag}\left(\bar{H}_{1}, \ldots, \bar{H}_{m}\right)$ with $\bar{H}_{i}=\left(k_{i 1} \ldots k_{i r_{i}-1}\right)$, $i=1, \ldots, m$. The second change of variables is meant to put the flow dynamics in normal form by re-defining the variable $\tilde{\eta}$ as $\tilde{\eta} \rightarrow \zeta=\tilde{\eta}-G_{\text {im }}(\tau) \bar{B}^{-1} \tilde{e}_{r}$. Finally, the state of the stabilizer unit of the regulator is changed as $\chi_{i} \rightarrow \tilde{\chi}_{i}=D_{g_{i}}\left(\chi_{i}-\operatorname{col}\left(\tilde{\xi}_{i}, \tilde{y}_{r_{i}}\right)\right)$, where $D_{g_{i}}=\operatorname{diag}\left(g^{r_{i}-1}, \ldots, g^{0}\right)$ for $i=1, \ldots, m$. The latter change of variables is motivated by the fact that the stabilizer unit is expected to estimate the state $\left(\tilde{\xi}, \tilde{y}_{r}\right)$ with a convergence rate that is tunable through the high-gain parameter $g$. After these coordinate transformations, the closed-loop error system reads as

$$
\begin{aligned}
\dot{\tau}= & 1, \quad \dot{\tilde{z}}=A_{11} \tilde{z}+A_{12} L \tilde{\xi}, \quad \dot{\tilde{\xi}}=\Lambda \tilde{\xi}+A_{23} \tilde{e}_{r} \\
\dot{\tilde{e}}_{r}= & \left(A_{33}+\bar{H} A_{23}+\bar{B} \Gamma_{\mathrm{im}}(\tau) G_{\mathrm{im}}(\tau) \bar{B}^{-1}-\kappa I_{m}\right) \tilde{e}_{r} \\
& +A_{31} \tilde{z}+\left(A_{32}-A_{33} \bar{H}+\bar{H} A_{22}-\bar{H} A_{23} \bar{H}\right) \tilde{\xi} \\
& -\kappa K D_{g}^{-1} \tilde{\chi}+\bar{B} \Gamma_{\mathrm{im}}(\tau) \zeta \\
\dot{\zeta}= & F_{\mathrm{im}}(\tau) \zeta+\left(F_{\mathrm{im}}(\tau) G_{\mathrm{im}}(\tau) \bar{B}^{-1}\right. \\
& \left.-G_{\mathrm{im}}(\tau) \bar{B}^{-1}\left(A_{33}+\bar{H} A_{23}\right)-\frac{d G_{\mathrm{im}}(\tau)}{d \tau} \bar{B}^{-1}\right) \tilde{e}_{r} \\
& -G_{\mathrm{im}}(\tau) \bar{B}^{-1} A_{31} \tilde{z}-G_{\mathrm{im}}(\tau) \bar{B}^{-1}\left(A_{32}-A_{33} \bar{H}\right. \\
& \left.+\bar{H} A_{22}-\bar{H} A_{23} \bar{H}\right) \tilde{\xi} \\
\dot{\tilde{\chi}}= & g H \tilde{\chi}+\Psi\left(\dot{\tilde{e}}_{r}-\overline{\dot{H}}\right)
\end{aligned}
$$


during flows, and

$$
\begin{aligned}
& \tau^{+}=0, \quad \tilde{z}^{+}=\tilde{z}, \quad \tilde{\xi}^{+}=\tilde{\xi}, \quad \tilde{e}_{r}^{+}=\tilde{e}_{r} \\
& \zeta^{+}=\Sigma_{\mathrm{im}} \zeta+\left(\Sigma_{\mathrm{im}} G_{\mathrm{im}}\left(\tau_{\max }\right)-G_{\mathrm{im}}(0)\right) \bar{B}^{-1} \tilde{e}_{r} \\
& \tilde{\chi}^{+}=\tilde{\chi}
\end{aligned}
$$

during jumps, where

$$
\begin{aligned}
\Lambda & =\operatorname{blkdiag}\left(S_{1}-B_{1} \bar{H}_{1}, \ldots, S_{m}-B_{m} \bar{H}_{m}\right) \\
H & =\operatorname{blkdiag}\left(H_{1}, \ldots, H_{m}\right), \Psi=\operatorname{blkdiag}\left(\Psi_{1}, \ldots, \Psi_{m}\right)
\end{aligned}
$$

with $H_{i}, i=1, \ldots, m$, appropriately defined Hurwitz matrices and $\Psi_{i} \in \mathbb{R}^{r_{i} \times 1}, i=1, \ldots, m$, the column vector that is all zeros except for the last element, which is one. For notational convenience in the equation for the $\dot{\tilde{\chi}}$ flow dynamics we have left $\dot{\tilde{e}}_{r}$ and $\dot{\tilde{\xi}}$, which should be replaced by their respective flow dynamics. For the forthcoming analysis it is just important to observe that those terms do not grow with $g$.

The final result can be concluded now by applying Proposition 5 in Appendix D.1 twice. In particular, we first pick $v_{1}=(\tilde{\xi}, \tilde{z}, \zeta), v_{2}=\tilde{e}_{r}$ (setting $\left.\tilde{\chi}=0\right)$, namely the system

$$
\begin{aligned}
\dot{v}_{1}= & \left(\begin{array}{ccc}
\Lambda & 0 & 0 \\
\star & A_{11} & 0 \\
\star & \star & F_{\mathrm{im}}(\tau)
\end{array}\right) v_{1}+\left(\begin{array}{c}
A_{23} \\
0 \\
\star
\end{array}\right) v_{2} \\
\dot{v}_{2}= & \left(A_{33}+\bar{H} A_{23}+\bar{B} \Gamma_{\mathrm{im}}(\tau) G_{\mathrm{im}}(\tau) \bar{B}^{-1}\right. \\
& \left.-\kappa I_{m}\right) v_{2}+(\star \star \star) v_{1}
\end{aligned}
$$

during flows, and

$$
v_{1}^{+}=\left(\begin{array}{ccc}
I & 0 & 0 \\
0 & I & 0 \\
0 & 0 & \Sigma_{\mathrm{im}}
\end{array}\right) v_{1}+\left(\begin{array}{c}
0 \\
0 \\
\star
\end{array}\right) v_{2}, v_{2}^{+}=v_{2}
$$

during jumps, with $\star$ denoting generic elements of no interest in the subsequent developments.

In order to satisfy the assumption for Proposition 5 we must verify that a Lyapunov function exists for the hybrid system

$$
\begin{aligned}
\dot{\tau} & =1, \quad \dot{\tilde{v}}_{1}=A_{1}(\tau) \tilde{v}_{1} \\
\tau^{+} & =0,
\end{aligned}
$$

where the definitions of $A_{1}(\tau)$ and $J_{1}$ are implied by (A.3)-(A.4) and the notation of Proposition 5. Toward this end, we first note that $A_{1}(\tau)$ is a block lower triangular matrix and $J_{1}$ is a block diagonal matrix. Furthermore, we observe that the Hybrid Internal Model
Property implies that the pair $\left(F_{\mathrm{im}}(\tau), \Sigma_{\mathrm{im}}\right)$ is chosen such that the origin of the system flowing as $\dot{\tau}=1$, $\dot{\tilde{\zeta}}=F_{\text {im }}(\tau) \tilde{\zeta}$ and jumping as $\tau^{+}=0, \tilde{\zeta}^{+}=\Sigma_{\text {im }} \tilde{\zeta}$ is GES. Since the matrix $\Lambda$ is Hurwitz by construction (by the choice of $K$ ) and $A_{11}$ is Hurwitz (by the minimumphase assumption), it is then easily verified that the set $\left[0, \tau_{\max }\right] \times\{0\}$ is GES for (A.5). From sampled-data systems results, this ensures that the eigenvalues of $\Phi_{1}\left(\tau_{\max }, 0\right) J_{1}$ are in the unit disk, where $\Phi_{1}\left(\tau_{\max }, 0\right)$ is the state transition matrix of the linear time-varying system $\dot{\bar{v}}_{1}=A_{1}(\tau) \bar{v}_{1}$, with $A_{1}(\tau)$ as in (A.5). This satisfies the condition needed for Proposition 5. With $-I_{m}$ clearly Hurwitz, and the parameters of the matrices in the dynamics bounded and continuous with respect to $\tau$, we can pick $\kappa>0$ large enough to make the origin of (A.3)-(A.4) GES.

Finally, we apply Proposition 5 again, but with $v_{1}=\left(\tilde{\xi}, \tilde{z}, \zeta, \tilde{e}_{r}\right), v_{2}=\tilde{\chi}$. We just ensured that the eigenvalues of the new " $\Phi_{1}\left(\tau_{\max }, 0\right) J_{1}$ " are inside the unit disk via the first implementation of Proposition 5. So, with $H$ Hurwitz, we can choose $g>0$ large enough to make the origin of (A.1)-(A.2) GES.

\section{B Proof of Proposition 3}

By bearing in mind (20), the fact that system (17) is state-output equivalent to system (30), and the choice of $\Gamma_{\mathrm{im}}(\tau)$, we prove the result by showing that the substates $\eta_{o}$ and $\eta_{n o}$ of the system

$$
\begin{aligned}
\dot{\eta}_{i} & =R_{o}^{T}(\tau) y_{\xi}, \quad \dot{\eta}_{o}=0, \quad \dot{\eta}_{n o}=0 \\
\eta_{i}^{+} & =0, \eta_{o}^{+}=\bar{N}_{o} \mathcal{G}_{o}^{-1} \eta_{i}+\bar{N}_{\text {ono }} \eta_{n o} \\
\eta_{n o}^{+} & =\bar{N}_{n o o} \mathcal{G}_{o}^{-1} \eta_{i}+\left(N_{n o}+K_{2} N_{\text {ono }}\right) \eta_{n o}
\end{aligned}
$$

asymptotically estimate the variables $\xi_{o}$ and $\xi_{n o}$ of (30). We first consider the $\eta_{i}$ variable whose hybrid dynamics immediately yield

$$
\begin{aligned}
\eta_{i}\left(\tau_{\max }\right) & =\int_{0}^{\tau_{\max }} R_{o}^{T}(\tau) R_{o}(\tau) d \tau \xi_{o}(0) \\
& =\mathcal{G}_{o} \xi_{o}(0)=\mathcal{G}_{o} \xi_{o}\left(\tau_{\max }\right)
\end{aligned}
$$

By defining the error coordinates as $\tilde{\eta}_{i}=\eta_{i}-\mathcal{G}_{o} \xi_{o}, \tilde{\eta}_{o}=$ $\eta_{o}-\xi_{o}, \tilde{\eta}_{n o}=\eta_{n o}-\xi_{n o}$, using the fact that $\xi_{o}\left(\tau_{\max }\right)=$ $\mathcal{G}_{o}^{-1} \eta_{i}\left(\tau_{\max }\right)$, the error system is represented by

$$
\begin{aligned}
\dot{\tilde{\eta}}_{i} & =R_{o}^{T}(\tau) R_{o}(\tau) \xi_{o}, \quad \dot{\tilde{\eta}}_{o}=0, \quad \dot{\tilde{\eta}}_{n o}=0 \\
\tilde{\eta}_{i}^{+} & =0, \tilde{\eta}_{o}^{+}=\bar{N}_{\text {ono }} \tilde{\eta}_{n o}, \tilde{\eta}_{n o}^{+}=\left(N_{n o}+K_{2} N_{\text {ono }}\right) \tilde{\eta}_{n o} .
\end{aligned}
$$

Using the fact that $\operatorname{eig}\left(N_{n o}+K_{2} N_{\text {ono }}\right) \in \mathcal{D}_{1}$ we immediately conclude that $\tilde{\eta}_{o}(t, j)$ and $\tilde{\eta}_{n o}(t, j)$ converge to zero. In particular, partitioning the change of variables 
$T(\tau)^{-1}$ and $\Upsilon$ introduced in Section 3.1 as $T(\tau)^{-1}=$ $\operatorname{col}\left(T_{o}(\tau)^{-1}, T_{n o}(\tau)^{-1}\right)$ and $\Upsilon=\operatorname{col}\left(\Upsilon_{o}, \Upsilon_{n o}\right)$ consistently with the partition $\varsigma=\operatorname{col}\left(\varsigma_{o}, \varsigma_{n o}\right)$, it turns out that the hybrid internal model property of Definition 2 is fulfilled with a $\Pi_{\eta}(\tau)$ of the form

$$
\Pi_{\eta}(\tau)=\left(\begin{array}{c}
\mathcal{G}_{o} T_{o}(\tau)^{-1} \\
T_{o}(\tau)^{-1} \\
\Upsilon_{o} T_{n o}(\tau)^{-1}+K_{2} T_{o}(\tau)^{-1}
\end{array}\right) .
$$

\section{Proof of Proposition 4}

By bearing in mind (20), the fact that system (17) is state-output equivalent to system (30), the choice of $\Gamma_{\operatorname{im}}(\tau)$, and the fact that $y_{w}=\mathbf{C} \chi_{o}$, we prove the result by showing that the system

$$
\dot{\eta}=F_{\mathrm{im}} \eta+G_{\mathrm{im}}(\tau) y_{\xi}, \quad \eta^{+}=\Sigma_{\mathrm{im}} \eta
$$

with $\eta=\operatorname{col}\left(\eta_{o}, \eta_{n o}\right), \eta_{o} \in \mathbb{R}^{m s_{o}}, \eta_{n o} \in \mathbb{R}^{s_{n o}^{\prime}}$, is an asymptotic observer of the $\left(\chi_{o}, \chi_{n o}\right)$ system (31)-(32).

Letting $\tilde{\eta}_{o}=\eta_{o}-\chi_{o}$ and $\tilde{\eta}_{n o}=\eta_{n o}-\chi_{n o}$, the error system reads as

$$
\begin{aligned}
\dot{\tilde{\eta}}_{o} & =\left(\mathbf{A}+\mathbf{K}_{1} \mathbf{C}\right) \tilde{\eta}_{o}, \quad \dot{\tilde{\eta}}_{n o}=0 \\
\tilde{\eta}_{o}^{+} & =\mathbf{M}_{o} \tilde{\eta}_{o}+\mathbf{M}_{\text {ono }} \tilde{\eta}_{n o} \\
\tilde{\eta}_{n o}^{+} & =\left(N_{n o}+K_{2} N_{\text {ono }}\right) \tilde{\eta}_{n o}+\mathbf{M}_{n o o} \tilde{\eta}_{o} .
\end{aligned}
$$

By rescaling the $\tilde{\eta}_{o}$ variable as $\tilde{\eta}_{o}^{\prime}=\mathbf{D}(\ell) \eta_{o}$, with $\mathbf{D}(\ell)=$ $\operatorname{blkdiag}(D(\ell), \ldots, D(\ell)), D(\ell)=\operatorname{diag}\left(1 \ell \ldots \ell^{s_{o}-1}\right)$, system (C.1) transforms as

$$
\begin{aligned}
\dot{\tilde{\eta}}_{o}^{\prime} & =\ell H \tilde{\eta}_{o}^{\prime}, \quad \dot{\tilde{\eta}}_{n o}=0 \\
\tilde{\eta}_{o}^{++} & =\mathbf{D}(\ell) \mathbf{M}_{o} \mathbf{D}^{-1}(\ell) \tilde{\eta}_{o}^{\prime}+\mathbf{D}(\ell) \mathbf{M}_{\text {ono }} \tilde{\eta}_{n o} \\
\tilde{\eta}_{n o}^{+} & =\left(N_{n o}+K_{2} N_{\text {ono }}\right) \tilde{\eta}_{\text {ono }}+\mathbf{N}_{n o o} \mathbf{D}^{-1}(\ell) \tilde{\eta}_{o}^{\prime}
\end{aligned}
$$

in which $H$ is Hurwitz. From this, using the fact that eig $\left(N_{\text {no }}+K_{2} N_{\text {ono }}\right) \in \mathcal{D}_{1}$, the result immediately follows by using Proposition 6 presented in Appendix D.2. In particular, partitioning the change of variables $T(\tau)^{-1}$ and $\Upsilon$ introduced in Section 3.1 as $T(\tau)^{-1}=\operatorname{col}\left(T_{o}(\tau)^{-1}, T_{n o}(\tau)^{-1}\right)$ and $\Upsilon=\operatorname{col}\left(\Upsilon_{o}, \Upsilon_{n o}\right)$ consistently with the partition $\varsigma=\operatorname{col}\left(\varsigma_{o}, \varsigma_{n o}\right)$, it turns out that the hybrid internal model property of Definition 2 is fulfilled with a $\Pi_{\eta}(\tau)$ of the form

$$
\Pi_{\eta}(\tau)=\left(\begin{array}{c}
\mathbf{P}(\tau) T_{o}(\tau)^{-1} \\
\Upsilon_{o} T_{n o}(\tau)^{-1}+K_{2} T_{o}(\tau)^{-1}
\end{array}\right)
$$

\section{Auxiliary Technical Results}

\section{D.1 High-Gain Stabilization of $\tau$-Dependent Hybrid Systems}

\section{Proposition 5 Consider the system}

$$
\begin{aligned}
& \left.\begin{array}{rl}
\dot{\tau} & =1, \dot{v}_{1}=A_{1}(\tau) v_{1}+B_{1}(\tau) v_{2} \\
\dot{v}_{2} & =A_{2}(\tau) v_{2}+B_{2}(\tau) v_{1}+\kappa H v_{2}
\end{array}\right\} \\
& \left(\tau, v_{1}, v_{2}\right) \in\left[0, \tau_{\max }\right] \times \mathbb{R}^{\rho} \times \mathbb{R}^{\sigma} \\
& \left.\tau^{+}=0, v_{1}^{+}=J_{1} v_{1}+L_{1} v_{2}\right\} \\
& v_{2}^{+}=J_{2} v_{2}+L_{2} v_{1} \\
& \left(\tau, v_{1}, v_{2}\right) \in\left\{\tau_{\max }\right\} \times \mathbb{R}^{\rho} \times \mathbb{R}^{\sigma}
\end{aligned}
$$

where $\tau_{\max }>0, H$ is Hurwitz, $B_{1}(\tau), B_{2}(\tau)$ and $A_{2}(\tau)$ are bounded with respect to $\tau$ and $A_{1}(\tau)$ is continuous with respect to $\tau$, over $\tau \in\left[0, \tau_{\max }\right]$. Furthermore, assume that the eigenvalues of $\Phi\left(\tau_{\max }, 0\right) J_{1}$ are within the unitary circle, where $\Phi\left(\tau, \tau_{0}\right)$ is the state transition matrix of the continuous-time system $\dot{\bar{v}}_{1}=A_{1}(\tau) \bar{v}_{1}$. There exists $\kappa^{*}>0$ such that, for each $\kappa \geq \kappa^{*}$, the $\operatorname{set}\left[0, \tau_{\max }\right] \times\{0\} \subset$ $\mathbb{R}^{\rho+\sigma+1}$ is globally exponentially stable.

Proof 1 The proof of the proposition is just sketched since it follows along the same lines of the proof of (Cox et al. 2014, Proposition 1), where the same result is proved with the matrices $A_{1}$ and $A_{2}$ not dependent on $\tau$.

We start by considering the system

$$
\begin{aligned}
& \left.\begin{array}{rl}
\dot{\tau} & =1 \\
\dot{v}_{1} & =A_{1}(\tau) v_{1}+B_{1}(\tau) v_{2}
\end{array}\right\} \quad\left(\tau, v_{1}\right) \in\left[0, \tau_{\max }\right] \times \mathbb{R}^{\rho} \\
& \left.\tau^{+}=0, v_{1}^{+}=J_{1} v_{1}+L_{1} v_{2}\right\} \quad\left(\tau, v_{1}\right) \in\left\{\tau_{\max }\right\} \times \mathbb{R}^{\rho}
\end{aligned}
$$

with state $\left(\tau, v_{1}\right)$ and input $v_{2}$. The Lyapunov function candidate for this system is

$$
W=\exp (-\epsilon \tau) v_{1}^{T} \Phi^{-1}\left(\tau, \tau_{\max }\right)^{T} X \Phi^{-1}\left(\tau, \tau_{\max }\right) v_{1},
$$

where $X=X^{T}>0$ and $\epsilon>0$. Arguments that are similar to those used in (Cox et al. 2014, Proposition 1) show that, across jumps, by appropriately choosing $X$ and $\epsilon$, the value of $W^{+}-W$ satisfies

$$
W^{+}-W \leq-d_{1}\left\|v_{1}\right\|^{2}+d_{2}\left\|v_{2}\right\|^{2},
$$

where $d_{1}$ and $d_{2}$ are positive scalar constants. Similarly, during flow, using

$$
\begin{aligned}
\frac{d \Phi^{-1}\left(\tau, \tau_{\max }\right)}{d \tau} & =-\Phi^{-1}\left(\tau, \tau_{\max }\right) \frac{d \Phi\left(\tau, \tau_{\max }\right)}{d \tau} \Phi^{-1}\left(\tau, \tau_{\max }\right) \\
& =-A_{1}(\tau)^{T} \Phi^{-1}\left(\tau, \tau_{\max }\right)^{T}
\end{aligned}
$$


it is possible to show that $\dot{W} \leq-c_{1}\left\|v_{1}\right\|^{2}+c_{2}\left\|v_{2}\right\|^{2}$ for some positive scalar constants $c_{1}, c_{2}$.

We now consider the system

$$
\begin{gathered}
\left.\dot{\tau}=1, \dot{v}_{2}=A_{2}(\tau) v_{2}+B_{2}(\tau) v_{1}+\kappa H v_{2}\right\} \\
\left(\tau, v_{2}\right) \in\left[0, \tau_{\max }\right] \times \mathbb{R}^{\sigma} \\
\left.\tau^{+}=0, v_{2}^{+}=J_{2} v_{2}+L_{2} v_{1}\right\}\left(\tau, v_{2}\right) \in\left\{\tau_{\max }\right\} \times \mathbb{R}^{\sigma}
\end{gathered}
$$

regarded as a system with state $\left(\tau, v_{2}\right)$ and input $v_{1}$. For this system we consider the candidate Lyapunov function $V=\exp (\lambda \tau) v_{2}^{T} P_{2} v_{2}$, where $P_{2}$ satisfies $-Q_{2}=$ $P_{2} H+H^{T} P_{2}$ with $Q_{2}>I>0$ and where $\lambda$ is a positive parameter. Again by the arguments used to prove (Cox et al. 2014, Proposition 1), it turns out that, during flows, the Lyapunov function satisfies

$$
\dot{V} \leq \exp (\lambda \tau)\left(\left(\lambda c_{3}+c_{4}-\kappa c_{5}\right)\left\|v_{2}\right\|^{2}+\frac{1}{\kappa} c_{6}\left\|v_{1}\right\|^{2}\right)
$$

for some positive $c_{i}, i=3, \ldots, 6$, while, during jumps,

$V^{+}-V \leq\left(d_{3}-\exp \left(\lambda \tau_{\max }\right) d_{4}\right)\left\|v_{2}\right\|^{2}+d_{5}\left\|v_{1}\right\|^{2}$

for some positive $d_{3}, d_{4}, d_{5}$, for all $\lambda>0$.

The result of the proposition then follows by considering the candidate Lyapunov function $\Psi=W+\ell V$, in which $\ell$ is a positive constant to be fixed. In particular (see (Cox et al. 2014, Proposition 1)), it is possible to show that, by taking $\ell$ sufficiently small, and $\lambda$ and $\kappa$ sufficiently large, we obtain $\Psi^{+}-\Psi \leq-\alpha_{1}\|v\|^{2}$ and $\dot{\Psi} \leq-\alpha_{2}\|v\|^{2}$ for some positive $\alpha_{1}$ and $\alpha_{2}$.

Standard Lyapunov arguments can then be used to conclude that the set $\left[0, \tau_{\max }\right] \times\{0\}$ is globally exponentially stable for (D.1).

\section{D.2 Polynomial Growth vs. Exponential Decay}

Proposition 6 Consider the system

$$
\left.\begin{array}{rl}
\dot{\tau} & =1, \\
\dot{v}_{1} & =A_{1} v_{1}, \dot{v}_{2}=\ell A_{2} v_{2}
\end{array}\right\}\left(\tau, v_{1}, v_{2}\right) \in\left[0, \tau_{\max }\right] \times \mathbb{R}^{\rho} \times \mathbb{R}^{\sigma}
$$

where $\tau_{\max }>0$, and the matrices $J_{2}(\ell), L_{1}(\ell)$ and $L_{2}(\ell)$ are polynomially dependent on $\ell$. If $A_{2}$ is Hurwitz and $J_{1} \exp \left(A_{1} \tau_{\max }\right) \in \mathcal{D}_{1}$, then there exists $\ell^{\star}>0$ such that, for each $\ell \geq \ell^{\star}$, the set $\left[0, \tau_{\max }\right] \times\{0\} \subset \mathbb{R}^{\rho+\sigma+1}$ is globally exponentially stable.
The proof of this proposition uses arguments that are similar to the ones used in the proof of Proposition 5 and it is thus omitted for reasons of space. The key difference is that the polynomial dependencies of the jump matrices require that the flow dynamics of the sub-states $v_{1}$ and $v_{2}$ do not affect each other. With this in mind, when the system in Proposition 6 is broken into sub-systems, as in the proof of Proposition D.1, the two Lyapunov function candidates are

$$
\begin{aligned}
W= & \exp (-\epsilon \tau) v_{1}^{T} \exp \left(A_{1}\left(\tau_{\max }-\tau\right)\right)^{T} \\
& \cdot X \exp \left(A_{1}\left(\tau_{\max }-\tau\right)\right) v_{1} \\
V= & \exp (\ell \lambda \tau) v_{2}^{T} P v_{2}
\end{aligned}
$$

with the notable difference being that $V$ has an exponential term that is dependent on $\ell$. 